\title{
OTFS: A New Modulation Scheme for High-Mobility
} Use Cases

\author{
M. K. Ramachandran ${ }^{1,2}$, G. D. Surabhi ${ }^{1}$ and A. Chockalingam ${ }^{1 *}$
}

\begin{abstract}
Among the several emerging use case families in $5 \mathrm{G}$, highmobility use case family is a technologically challenging one. It is expected that there will be a growing demand for mobile services in vehicles, high-speed trains, and even aircraft. The degree of mobility support required (i.e., speed) will depend upon the specific use case (e.g., $500 \mathrm{~km} / \mathrm{h}$ in bullet trains and $1000 \mathrm{~km} / \mathrm{h}$ in airplanes). Mobility-ondemand, ranging from very high mobility to low or no mobility, need to be supported. The currently used waveforms fail to perform well in highmobility scenarios where the Doppler shifts witnessed are quite high (e.g., several kHz of Doppler). Orthogonal time-frequency space (OTFS) is a recently proposed radio access technology waveform suited very well for high-mobility environments. It is a two-dimensional modulation scheme in which information symbols are multiplexed in the delay-Doppler domain. We present an overview of delay-Doppler representation of wireless channels and introduce OTFS modulation along with OTFS basis functions. We illustrate the slow variability and sparse nature of the delay-Doppler channel using an urban multi-lane scenario. Focusing on MIMO-OTFS systems, we present signal detection and channel estimation schemes and their performance. MIMO-OTFS is shown to achieve significantly better performance compared to MIMO-OFDM in high-Doppler environments operating in $4 \mathrm{GHz}$ and $28 \mathrm{GHz}$ frequency bands.
\end{abstract}

Keywords: High-mobility use cases, OTFS modulation, Delay-Doppler domain, MIMO-OTFS, mmWave communication, Signal detection, Channel estimation

\section{Introduction}

Next-generation wireless networks (including $5 \mathrm{G}$ networks) are expected to see the emergence of several new use cases and business models driven by the emerging needs of users and system operators. This will be enabled by the maturity of current technologies and emergence of new technologies. The variety of applications and the corresponding performance requirements in these scenarios will be vastly different. Several use case families such as broadband access everywhere (e.g., pervasive video), extreme real-time communications (e.g., tactile internet with sub-millisecond latency), ultra-reliable communications (e.g., e-health services), massive internet of things (e.g., sensor networks, smart wearables), and high mobility (e.g., high-speed trains, airplanes) are being envisaged ${ }^{1}$. In particular, the high-mobility use case family is technologically challenging. Mobility requirements are expressed in terms of relative speed between the user and the network edge at which consistent user experience must be ensured. For example, in-vehicle mobile broadband service requires a mobility support of up to $500 \mathrm{~km} / \mathrm{h}$ for bullet trains and $1000 \mathrm{~km} / \mathrm{h}$ for airplanes. Wireless channels in such high-mobility scenarios are doubly selective, where multipath effects result in inter-symbol interference (due to frequency selectivity) and Doppler shifts (due to time selectivity). High Doppler shifts make the
${ }^{1}$ Department of ECE, Indian Institute of Science, Bangalore 560012, India. 2 Present Address: Qualcomm India Private Limited, Bangalore 560066, India. *achockal@iisc.ac.in 
channel highly time selective ${ }^{2}$. The currently used waveforms fail to perform well in high-mobility scenarios where the Doppler shifts encountered are high (e.g., several kHz of Doppler). For example, orthogonal frequency division multiplexing (OFDM)-based systems, while resilient to inter-symbol interference (ISI) ${ }^{3}$, suffer from performance degradation due to inter-carrier interference (ICI) caused by high Doppler shifts ${ }^{4}$. Pulse shaping is one approach to jointly mitigate ISI and ICI in OFDM systems ${ }^{5-7}$. This approach uses time-frequency domain and optimal pulse shapes for the waveform design. However, pulse shaping is not very effective in handling timeselectivity in high-mobility channels. Another approach in the literature makes use of canonical decomposition of the received signal into delay and Doppler shifted versions of a basis signal, and proposes a delay-Doppler RAKE receiver that offers joint multipath-Doppler diversity ${ }^{8}$. Also, time-selective signaling schemes with overlapping symbols can provide better performance despite ISI, if properly designed. Such schemes that spread the information over time have been studied in the context of CDMA ${ }^{9,10}$. The above approaches differ from OTFS modulation in that OTFS is designed in delay-Doppler domain rather than in time-frequency domain. OTFS uses delay-Doppler domain for channel representation as well as multiplexing of information symbols. This difference between OTFS and the approach in ${ }^{8}$ has been pointed out in ${ }^{13}$.

Orthogonal time-frequency space (OTFS) modulation, proposed by Hadani et al. in WCNC'2017 ${ }^{11}$, is a multiplexing scheme suited well for doubly-selective channels. This twodimensional (2D) modulation scheme uses an approach where multiplexing of information symbols happens in the delay-Doppler domain ${ }^{11-14}$. This is different from conventional modulation approaches (e.g., OFDM), where multiplexing is done in the time-frequency domain. OTFS basis functions (waveforms) have strong resilience to delay-Doppler shifts imparted by the channel ${ }^{12}$. OTFS has been shown to achieve significant performance gains compared to OFDM in high-Doppler channels with vehicular speeds of $500 \mathrm{~km} / \mathrm{h}$ in $4 \mathrm{GHz}$ band and $40 \mathrm{~km} / \mathrm{h}$ in $28 \mathrm{GHz}$ band ${ }^{11,15,16}$. In the delay-Doppler domain (signaling domain for OTFS), the basis functions for OTFS are 2D localized pulses. The information symbols are multiplexed using these 2D pulses (basis functions). The idea is to use a transformation that relates the information symbols and the wireless channel in the delay-Doppler domain. Also, when viewed in the delay-Doppler domain, the doubly selective channel is sparse and slowly varying. OTFS modulation can be implemented using the existing multicarrier modulation schemes (such as OFDM) using additional pre- and post-processing $2 \mathrm{D}$ transforms.

Several recent works on OTFS have emerged in the literature recognizing the suitability of OTFS waveform for next-generation wireless systems including mmWave communication systems ${ }^{15-36}$. These works have focused on vectorized input-output relation in OTFS, schemes for equalization/detection and channel estimation, diversity order achieved by OTFS, peak-toaverage power ratio (PAPR) characteristics, pulse shaping, effect of oscillator phase noise, exploitation of the effective channel structure for lowcomplexity linear detection, space-time coded OTFS, multiple access using OTFS, and relation between OTFS and generalized frequency division multiplexing (GFDM). In this paper, we present an overview of delay-Doppler representation of wireless channels and introduce OTFS modulation along with OTFS basis functions. We illustrate the slow variability and sparse nature of the delay-Doppler channel using an urban multilane scenario. Focusing on MIMO-OTFS systems ${ }^{26}$, we present signal detection and channel estimation schemes and their performance. MIMOOTFS is shown to achieve significantly better performance compared to MIMO-OFDM in high-Doppler environments operating in $4 \mathrm{GHz}$ and $28 \mathrm{GHz}$ frequency bands. For example, in a $2 \times 2$ MIMO-OTFS system, a bit error rate (BER) of $10^{-5}$ is achieved at a signal-to-noise ratio (SNR) of $14 \mathrm{~dB}$ for a Doppler of $1850 \mathrm{~Hz}(500$ $\mathrm{km} / \mathrm{h}$ speed at $4 \mathrm{GHz}$ ), whereas the performance of MIMO-OFDM in the same system floors at a BER of 0.02. A similar performance advantage of MIMO-OTFS over MIMO-OFDM is witnessed in mmWave frequency band (e.g., $28 \mathrm{GHz}$ ) as well.

The rest of the paper is organized as follows. The delay-Doppler domain, its characteristics, and its connection to OTFS modulation are presented in Sect. 2. The OTFS modulation is presented in Sect. 3. The MIMO-OTFS system model and a vectorized formulation for its input-output relation are presented in Sect. 4. MIMO-OTFS signal detection using message passing algorithm and the BER performance are presented in Sect. 5. The delay-Doppler channel estimation scheme and the resulting performance are presented in Sect. 6. Conclusions are presented in Sect. 7. 

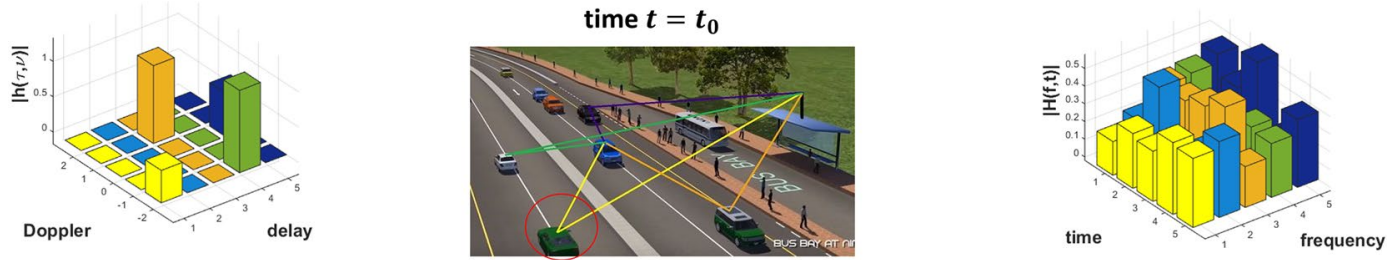

time $t=t_{0}+\Delta$
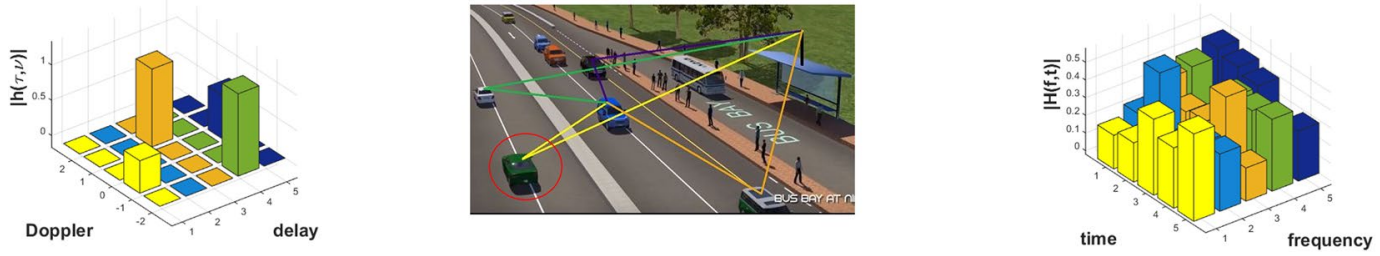

Figure 1: Example of a wireless channel in an urban multi-lane scenario illustrating the sparsity and slow variability of the channel in the delay-Doppler representation.

\section{The Delay-Doppler Domain and OTFS Modulation}

In this section, we discuss the delay-Doppler representation of wireless channels and its connection to OTFS modulation. We first illustrate the sparsity and slow variability of the delay-Doppler representation of wireless channels and then discuss its connections to OTFS modulation.

\subsection{Delay-Doppler Representation of Wireless Channels}

Different representations can be used for modeling the impulse response of a linear time-varying multipath channel depending upon the parameters (independent variables) chosen. Denoting time, frequency, delay, and Doppler variables with $t, f, v$, and $\tau$, respectively, the impulse response of a time-varying channel can be expressed as a function of time-frequency $H(t, f)$, time-delay $h(t, \tau)$, and delay-Doppler $h(\tau, v)$. In the time-frequency (i.e., $H(t, f)$ ) and time-delay (i.e., $h(t, \tau))$ representations of the channel, channel coefficients vary with time at a rate $(\propto 1 /$ coherence time $)$ which depends on the mobility and the operating frequency. This causes the channel to change rapidly, rendering channel estimation difficult. A more compact and equivalent representation of the channel is the delay-Doppler domain impulse response $h(\tau, v)^{11,14}$. The channel coefficients (taps) in this representation correspond to a group of reflectors with a specific delay value depending on reflectors' relative distance and Doppler value depending on reflectors' relative velocity with the transmitter and receiver. The velocity and the distance remain roughly constant for at least a few milliseconds. Hence, the channel in the delay-Doppler domain appears time invariant for a longer observation duration as compared to that in the time-frequency representation ${ }^{14}$. Besides time invariance, the delayDoppler representation of the channel impulse response results in a sparse representation of the time-varying channel, thus requiring only fewer channel parameters to be estimated. The slow variability and sparse nature of the delay-Doppler channel can be visualized in Figs. 1 and 2 .

Figure 1 shows an example of a scenario encountered in urban multi-lanes. In the figure, the transmitter mounted on top of the bus bay transmits a signal which is intended to be received by one of the moving cars (blue car in the figure). The transmitted signal gets reflected by multiple mobile reflectors (other cars) and reaches the receiver. For the sake of illustration, we have considered four paths corresponding to four reflectors. The paths corresponding to different reflectors are indicated with different colors. The impulse response of the wireless channel in this scenario is illustrated in both delay-Doppler domain as well as time-frequency domain in the same figure at time $t=t_{0}$ and $t=t_{0}+\Delta$. From the figure, it can be seen that the channel representation in the delay-Doppler domain is very sparse compared to that in time-frequency representation. Also, since the channel coefficients in the delay-Doppler representation correspond to a group of reflectors with a particular delay and Doppler shift, we see four non-zero channel coefficients corresponding to the four reflectors in 


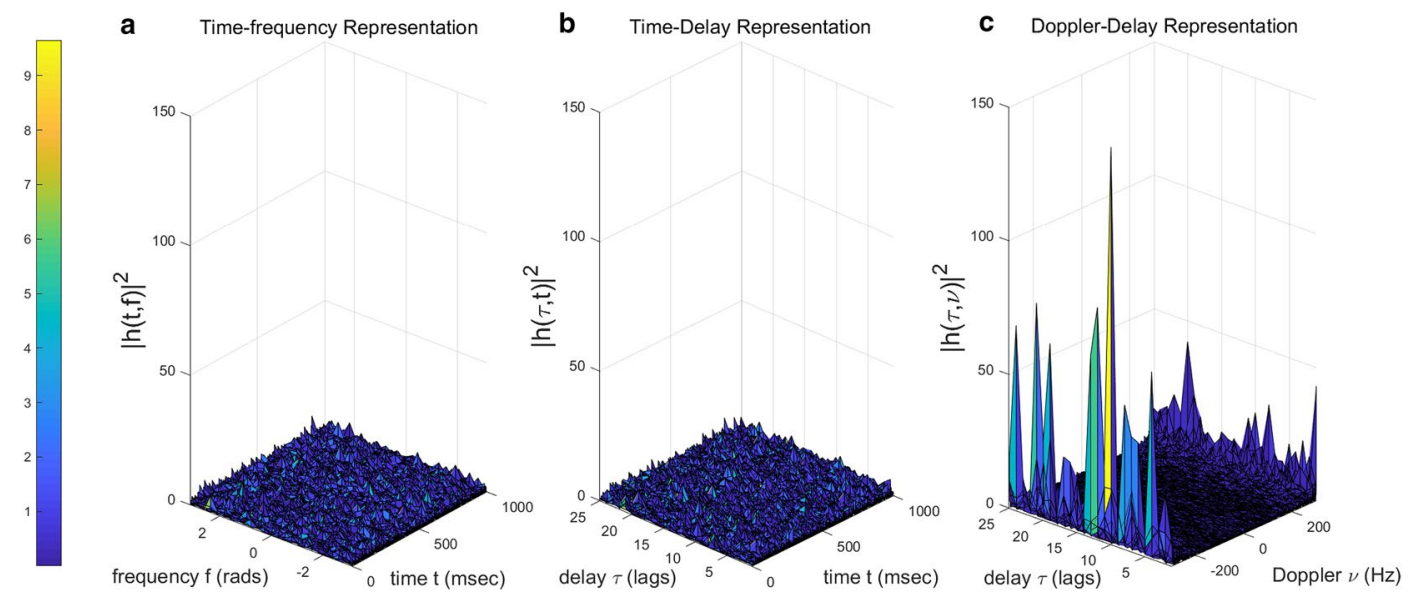

Figure 2: Squared magnitude of the impulse response of a $300 \mathrm{~Hz}$ Jakes Doppler channel model with 25 uniform power delay profile taps in a time-frequency domain, b time-delay domain, and c Doppler-delay domain.

the figure. This illustrates that the delay-Doppler representation of the channel impulse response is sparse and captures the geometry of the wireless environment. Figure 1 also illustrates the channel at time $t=t_{0}+\Delta$, in which one of the reflectors (green car which is circled) has decelerated. Due to the deceleration (decrease in the speed) of one of the reflectors, the coefficient corresponding to this reflector now falls in a different bin on the Doppler axis (yellow bar) in the delay-Doppler grid, whereas other coefficients remain in their bins at time $t_{0}$. However, the time-frequency representation of the channel shows significant variation at times $t_{0}$ and $t_{0}+\Delta$. This illustrates the slow variability of the channel in the delay-Doppler representation compared to time-frequency representation.

Figure 2 shows the squared magnitude of the impulse response of Jakes channel model with maximum Doppler of $300 \mathrm{~Hz}^{2}$ with 25 delay taps and uniform power delay profile; (a) in time-frequency domain, (b) in time-delay domain which is related by Fourier transform with the timefrequency domain along the delay axis, and (c) in delay-Doppler domain which is related to the time-frequency domain by a transform called $2 \mathrm{D}$ symplectic Fourier transform ${ }^{14}$. As observed from Fig. 2, the impulse response is spread in the time-frequency and time-delay representations, whereas it is peaky (localized) in a few delayDoppler bins in the delay-Doppler representation, i.e., the delay-Doppler impulse response is more sparse. The received signal $y(t)$ in the delayDoppler representation is the sum of reflected copies of the transmitted signal $x(t)$, which are time delayed $(\tau)$ and frequency $\operatorname{shifted}(\nu)$ by the reflectors. Thus, the channel-symbol coupling/ interaction in this domain is given by the following double integral:

$$
y(t)=\int_{v} \int_{\tau} h(\tau, v) x(t-\tau) e^{j 2 \pi \nu(t-\tau)} \mathrm{d} \tau \mathrm{d} \nu .
$$

\subsection{OTFS: Modulation in the Delay- Doppler Domain}

Fundamentally, a signal can be represented either as a superposition of delta functions (called the temporal/time representation) or as a superposition of complex exponential functions (called the frequency representation). These two representations are one-dimensional (1D) and are interchangeable through Fourier transform. Apart from the time and frequency domain representations, a signal can also be represented as a quasi-periodic function of delay and Doppler 12. The delay-Doppler signal representation is a two-dimensional (2D) representation which can be converted to the time and frequency representations through the Zak transforms $Z_{t}$ and $Z_{f}$, respectively. The signals in the delay-Doppler domain can be viewed as functions $\phi(\tau, \nu)$ on a $2 \mathrm{D}$ delay-Doppler plane whose points are parameterized by $\tau$ and $\nu$. This representation is a quasiperiodic representation and has an associated delay period $\tau_{r}$ and a Doppler period $v_{r}$, such that $\tau_{r} v_{r}=1$. The delay-Doppler representation is related to time and frequency representations by Zak transforms $Z_{t}$ and $Z_{f}$, respectively, given by ${ }^{12}$ 


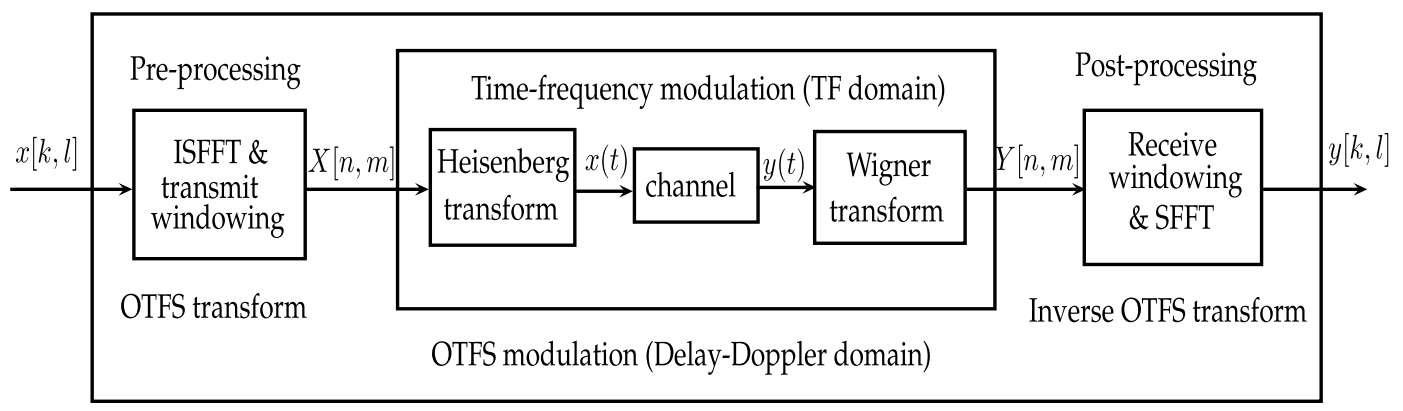

Figure 3: OTFS modulation scheme.

$Z_{t}(\phi)=\int_{0}^{v_{r}} e^{j 2 \pi t v} \phi(t, v) \mathrm{d} v$

$Z_{f}(\phi)=\int_{0}^{\tau_{r}} e^{-j 2 \pi \tau f} \phi(\tau, f) \mathrm{d} \tau$.

Quasi-periodic signals are periodic up to a multiplicative phase, i.e., $\phi\left(\tau+n \tau_{r}, v+m v_{r}\right)=$ $e^{j 2 \pi\left(n v \tau_{r}-m \tau v_{r}\right)} \phi(\tau, \nu)$. It is noted that the quasiperiodicity property is necessary to recover the $2 \mathrm{D}$ function $\phi(\tau, v)$ uniquely from its $1 \mathrm{D}$ projection $Z_{t}(\phi)$ or $Z_{f}(\phi)$. Without it, a onedimensional signal will admit infinitely many delay-Doppler representations ${ }^{12}$. The delayDoppler signal representation is not unique because $\tau_{r}$ and $v_{r}$ can take any value such that $\tau_{r} v_{r}=1$. When the limit $\tau_{r}$ tends to infinity and $v_{r}$ tends to zero, the delay-Doppler representation becomes the time representation. Similarly, when the limit $v_{r}$ tends to infinity and $\tau_{r}$ tends to zero, the delay-Doppler representation becomes the frequency representation of the signal.

A fundamental feature of OTFS modulation that distinguishes it from other time-frequency (TF) modulation schemes is the use of delayDoppler domain for multiplexing the modulation symbols. The symbols in the delay-Doppler domain can be converted into temporal domain for transmission using the Zak transform $Z_{t}$. The transformation that uses a single Zak transform to convert a signal in delay-Doppler domain to a signal in time domain can also be carried out in two steps. That is, the signal is first transformed to time-frequency domain using a $2 \mathrm{D}$ inverse symplectic finite Fourier transform, and then the resulting time-frequency signal is converted to a temporal representation using a Heisenberg transform. The two-step implementation used to transform the symbols in the delay-Doppler domain to time domain signal facilitates the implementation of OTFS modulation using simple pre- and post-processing steps over any multicarrier modulation scheme such as OFDM. The series of transformations involved in OTFS modulation and demodulation transforms a rapidly time-varying multipath channel into a slowly varying delay-Doppler domain channel, which is also sparse. The sparsity in the effective channel in OTFS modulation can be exploited to estimate only a few channel parameters compared to that in the time-frequency domain. Also, since the channel in the delay-Doppler domain is slowly varying, the channel can be estimated less frequently, which can reduce the overhead on channel estimation in a rapidly time-varying channel. A detailed description of the OTFS modulation scheme architected using pre- and post-processing operations over a multicarrier modulation is presented in Sect. 3.2.

\section{OTFS Modulation}

OTFS modulation can be implemented using preand post-processing to existing OFDM-based multicarrier modulation schemes. This is done using as a series of $2 \mathrm{D}$ transformations at both the transmitter and receiver. Figure 3 shows the block diagram of the OTFS modulation scheme. The inner box in the diagram shows the familiar multicarrier modulation in the time-frequency domain, ${ }^{1}$ and the outer box with a pre-processor and a post-processor block implements the OTFS modulation in the delay-Doppler domain. The information symbols $x[k, l]$ (e.g., QAM symbols) residing in the delay-Doppler domain are first transformed to the familiar time-frequency (TF) domain signal $X[n, m]$ using the $2 \mathrm{D}$ inverse symplectic finite Fourier transform (ISFFT) and a 2D windowing is applied. The Heisenberg transform

\footnotetext{
${ }^{1}$ Multicarrier modulation systems such as OFDM or filtered OFDM can constitute the inner core of OTFS modulation. For example, recently, it has been shown in ${ }^{25}$ that OTFS can be implemented using a GFDM framework and that OTFS achieves better performance compared to GFDM.
} 
is then applied to the TF signal $X[n, m]$ to transform to the temporal domain signal $x(t)$ for transmission. The received signal $y(t)$ at the receiver is transformed back to a TF signal $Y[n, m]$ through inverse Heisenberg transform (also called Wigner transform). $Y[n, m]$ thus obtained is transformed to the delay-Doppler domain signal $y[k, l]$ using a $2 \mathrm{D}$ receive windowing and the symplectic finite Fourier transform (SFFT), for demodulation.

In the following subsections, we describe the signal models in TF modulation and OTFS modulation. Let $T$ denote the TF modulation symbol time and $\Delta f$ denote the subcarrier spacing. Let $x[k, l], k=0, \ldots, N-1, l=0, \ldots, M-1$ denote the information symbols transmitted in a given packet burst. Let $W_{t x}[n, m]$ and $W_{r x}[n, m]$ denote the transmit and receive windows, respectively.

\subsection{Time-Frequency Modulation}

- Let $\varphi_{t x}(t)$ and $\varphi_{r x}(t)$ denote the transmit and receive pulses, respectively. We assume $\varphi_{t x}(t)$, $\varphi_{r x}(t)$ to be ideal pulses satisfying the biorthogonality property with respect to translations by integer multiples of time $T$ and frequency $\Delta f$, i.e.,

- $\int e^{-j 2 \pi m \Delta f(t-n T)} \varphi_{r x}^{*}(t-n T) \varphi_{t x}(t) \mathrm{dt}=\delta(\mathrm{m}) \delta(\mathrm{n})$.

The bi-orthogonality property of the pulse shapes eliminates the cross-symbol interference at the receiver. Although ideal pulses are not practically realizable, they can be approximated by the pulses whose support is highly concentrated in time and frequency ${ }^{18}$, given the constraint imposed by the uncertainty principle. ${ }^{2}$ The signal in the TF domain $X[n, m], n=0, \ldots, N-1, m=0, \ldots, M-1$ is transmitted in a given packet burst.

- TF modulation/Heisenberg transform The signal in the time-frequency domain $X[n, m]$ is transformed to the time domain signal $x(t)$ using the Heisenberg transform given by

- $x(t)=\sum_{n=0}^{N-1} \sum_{m=0}^{M-1} X[n, m] \varphi_{t x}(t-n T) e^{j 2 \pi m \Delta f(t-n T)}$.

- TF demodulation/Wigner transform At the receiver, the time domain signal is trans-

\footnotetext{
2 Design and performance of OTFS systems using practical pulse shapes have been discussed in ${ }^{24}$. It has been shown in ${ }^{24}$ that the OTFS modulation with practical pulse shapes like rectangular pulse and prolate spheroidal waveform shows better error performance compared to the conventional OFDM.
}

formed back to the TF domain using Wigner transform given by

- $Y[n, m]=\left.A_{\varphi_{r x}, y}(\tau, \nu)\right|_{\tau=n T, v=m \Delta f}$,

where $A_{\varphi_{r x}, y}(\tau, \nu)$ is the cross ambiguity function

$$
A_{\varphi_{r x}, y}(\tau, v)=\int \varphi_{r x}^{*}(t-\tau) y(t) e^{-j 2 \pi v(t-\tau)} \mathrm{dt}
$$

and $y(t)$ is related to $x(t)$ by (1). The relation between $Y[n, m]$ and $X[n, m]$ for TF modulation can be derived as ${ }^{11}$

$$
Y[n, m]=H[n, m] X[n, m]+V[n, m],
$$

where $V[n, m]$ is the additive white Gaussian noise and $H[n, m]$ is given by

$$
H[n, m]=\int_{\tau} \int_{\nu} h(\tau, \nu) e^{j 2 \pi v n T} e^{-j 2 \pi(\nu+m \Delta f) \tau} \mathrm{d} \nu \mathrm{d} \tau .
$$

\subsection{OTFS Modulation}

- When OTFS modulation is implemented as pre- and post-processing to TF modulation, the delay-period $\tau_{r}$ and Doppler period $v_{r}$ are chosen as

$$
\text { - } \quad\left(\tau_{r}, v_{r}\right)=\left(\frac{1}{\Delta f}, \frac{1}{T}\right) .
$$

- Let $X_{p}[n, m]$ be the periodized version of $X[n, m]$ with period $(N, M)$. The SFFT of $X_{p}[n, m]$ is given by

$$
\text { - } x_{p}[k, l]=\sum_{n=0}^{N-1} \sum_{m=0}^{M-1} X_{p}[n, m] e^{-j 2 \pi\left(\frac{n k}{N}-\frac{m l}{M}\right)},
$$

and the ISFFT is $X_{p}[n, m]=\operatorname{SFFT}^{-1}\left(\mathrm{x}_{\mathrm{p}}[\mathrm{k}, 1]\right)$, given by

$X_{p}[n, m]=\frac{1}{M N} \sum_{k=0}^{N-1} \sum_{l=0}^{M-1} x_{p}[k, l] e^{j 2 \pi\left(\frac{n k}{N}-\frac{m l}{M}\right)}$.

- Information symbols $x_{p}[k, l]$, $k=0, \ldots, N-1, l=0, \ldots, M-1$, are transmitted in a given packet burst.

- OTFS transform/pre-processing The information symbols in the delay-Doppler domain are mapped to TF domain symbols $X[n, m]$ as

- $X[n, m]=W_{t x}[n, m] \operatorname{SFFT}^{-1}\left(\mathrm{x}_{\mathrm{p}}[\mathrm{k}, 1]\right)$,

where $W_{t x}[n, m]$ is the square summable transmit windowing function. 
- $X[n, m]$ thus obtained is in the TF domain and it is TF modulated as described in the previous subsection, and $Y[n, m]$ is obtained by (6).

- OTFS demodulation/post-processing A receive window $W_{r x}[n, m]$ is applied to $Y[n, m]$ and periodized to obtain $Y_{p}[n, m]$ which has the period $(N, M)$, as

- $Y_{W}[n, m]=W_{r x}[n, m] Y[n, m]$,

$$
Y_{p}[n, m]=\sum_{k, l=-\infty}^{\infty} Y_{W}[n-k N, m-l M] .
$$

The symplectic finite Fourier transform is then applied to $Y_{p}[n, m]$ to convert it from TF domain back to delay-Doppler domain $y_{p}[k, l]$, as

$$
y_{p}[k, l]=\operatorname{SFFT}\left(\mathrm{Y}_{\mathrm{p}}[\mathrm{n}, \mathrm{m}]\right) .
$$

The output sequence of demodulated symbols is obtained as $y[k, l]=y_{p}[k, l]$ for $k=0,1, \ldots, N-1$ and $l=0,1, \ldots, M-1$.

The input-output relation in OTFS modulation can be derived as ${ }^{11}$

$$
\begin{aligned}
y[k, l]= & \frac{1}{M N} \sum_{m=0}^{M-1} \sum_{n=0}^{N-1} x[n, m] \\
& h_{w}\left(\frac{k-n}{N T}, \frac{l-m}{M \Delta f}\right)+v[k, l],
\end{aligned}
$$

where $v[k, l]$ is the additive white Gaussian noise and

$h_{w}\left(\frac{k-n}{N T}, \frac{l-m}{M \Delta f}\right)=\left.h_{w}\left(v^{\prime}, \tau^{\prime}\right)\right|_{v^{\prime}=\frac{k-n}{N T}, \tau^{\prime}=\frac{l-m}{M \Delta f}}$,

where $h_{w}\left(v^{\prime}, \tau^{\prime}\right)$ is the circular convolution of the channel response with a windowing function $w(\tau, \nu)$, given by

$h_{w}\left(v^{\prime}, \tau^{\prime}\right)=\int_{v} \int_{\tau} h(\tau, v) w\left(v^{\prime}-v, \tau^{\prime}-\tau\right) \mathrm{d} \tau \mathrm{d} v$,

where $w(\tau, \nu)$ is given by

$$
\begin{aligned}
w(\tau, v)= & \sum_{m=0}^{M-1} \sum_{n=0}^{N-1} W_{t x}[n, m] \\
& W_{r x}[n, m] e^{-j 2 \pi(v n T-\tau m \Delta f)} .
\end{aligned}
$$

Note that the window $w(\tau, v)$ in $(17)$ is a 2D Dirichlet kernel introduced due to the finite transmission bandwidth and finite transmission time interval of the signal. Any function convolved with a Dirichlet kernel (as in 17) is a Fourier series (2D in this case) approximation to the function itself. The error in approximation depends on the support of the window function. Thus, the transmit and the receive window functions used allow the finite 2D summation approximation of the input-output relation in OTFS modulation.

\subsubsection{OTFS Basis Functions}

The OTFS basis functions in the delay-Doppler domain, time-frequency domain, and time domain for different delay $(l)$ and Doppler $(k)$ indices are shown in Figs. 4, 5 and 6. For this illustration of basis functions, we have chosen a frame size of $M=32, N=32$, and a subcarrier spacing of $\Delta f=15 \mathrm{kHz}$. We see that, in the TF domain, the basis function spans the entire time-frequency plane. This resembles spread spectrum. The time domain waveform is a train of pulses modulated by a tone ${ }^{12}$. Locally, the waveform resembles TDM pulse (localized pulse in time domain), and globally, the shape of the train resembles a tone or FDM pulse (localized pulse in frequency domain).

Figure 7 shows the OTFS basis functions (carrier waveforms) in the time domain for Doppler and delay indices $(k, l)=(0,0),(k, l)=(0,15)$, and $(k, l)=(2,0)$. We see that, as the Doppler index $(k)$ changes, the frequency of the pulse train changes (as in FDM). Similarly, we also note that, as the delay index $(l)$ changes, the position of the pulses gets shifted in time (as in TDM). This illustrates that the OTFS waveform is a generalization of TDM and FDM.

\subsection{Vectorized Formulation of the Input- Output Relation}

Consider a channel with $P$ signal propagation paths (taps). Let the $i$ th path be associated with a delay value $\tau_{i}$, a Doppler value $v_{i}$, and a fade coefficient $h_{i}$. The delay-Doppler channel impulse response can be written as

$h(\tau, v)=\sum_{i=1}^{P} h_{i} \delta\left(\tau-\tau_{i}\right) \delta\left(v-v_{i}\right)$

Assume that the transmit and receive windows used in modulation, $W_{t x}[n, m]$ and $W_{r x}[n, m]$, are rectangular. Define $\tau_{i}=\frac{\alpha_{i}}{M \Delta f}$ and $v_{i}=\frac{\beta_{i}}{N T}$, where

$\alpha_{i}$ and $\beta_{i}$ are integers denoting the indices of the delay tap (with delay $\tau_{i}$ ) and Doppler tap (with Doppler value $v_{i}$ ). In practice, although the delay and Doppler values are not exactly integer multiples of the taps, they can be well approximated by a few delay-Doppler taps in the discrete domain ${ }^{40}$. With the above assumptions, the input-output relation for the channel in (19) can be derived as ${ }^{18}$ 
Delay-Doppler domain

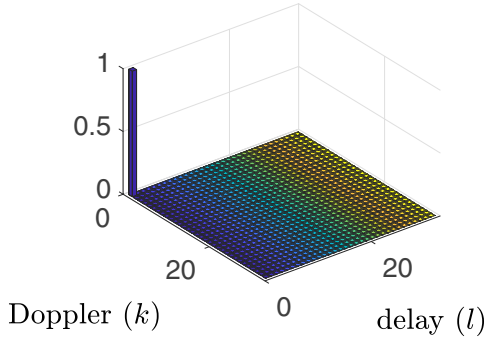

$k=0, l=0$

Time-frequency domain

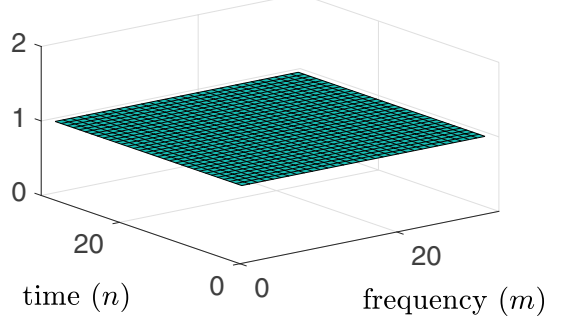

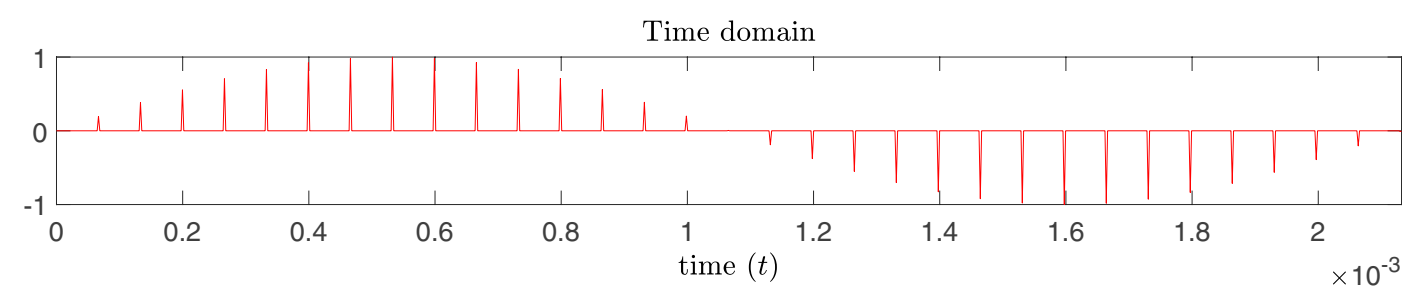

Figure 4: OTFS basis functions in delay-Doppler domain, time-frequency domain, and time domain for Doppler index $k=0$ and delay index $I=0$.

Delay-Doppler domain $\quad k=2, l=0$

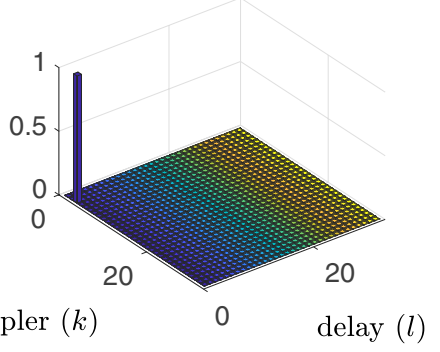

Time-frequency domain

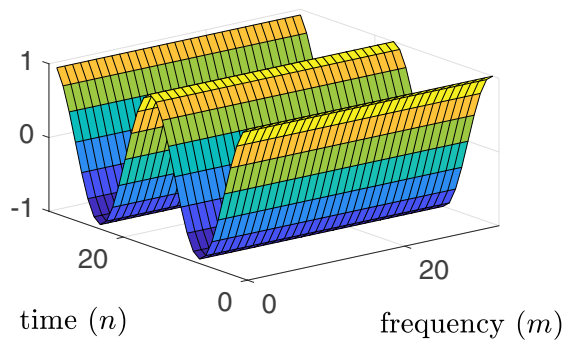

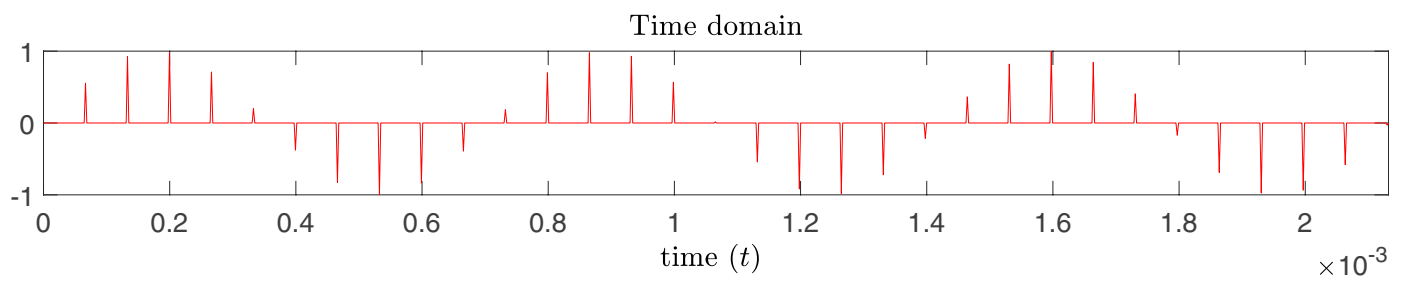

Figure 5: OTFS basis functions in delay-Doppler domain, time-frequency domain, and time domain for Doppler index $k=2$ and delay index $/=0$.

$$
y[k, l]=\sum_{i=1}^{P} h_{i}^{\prime} x\left[\left(k-\beta_{i}\right)_{N},\left(l-\alpha_{i}\right)_{M}\right]+v[k, l],
$$

where

$$
h_{i}^{\prime}=h_{i} e^{-j 2 \pi v_{i} \tau_{i}} .
$$

It is assumed that the $h_{i}$ s are i.i.d and are distributed as $\mathcal{C N}(0,1 / P)$, assuming uniform scattering profile. Denoting the $N \times M$ channel matrix in the delay-Doppler grid by $\widehat{\mathbf{H}}$, the $(k, l)$ th entry of $\widehat{\mathbf{H}}$, denoted by $h(k, l)$, is defined as

$$
\hat{h}(k, l)= \begin{cases}h_{i}^{\prime} \text { if } k=\beta_{i} \& l=\alpha_{i} \text { for some } i \in\{1,2, \ldots, P\} \\ 0 \text { otherwise. }\end{cases}
$$


Delay-Doppler domain $\quad k=2, l=2$

Time-frequency domain
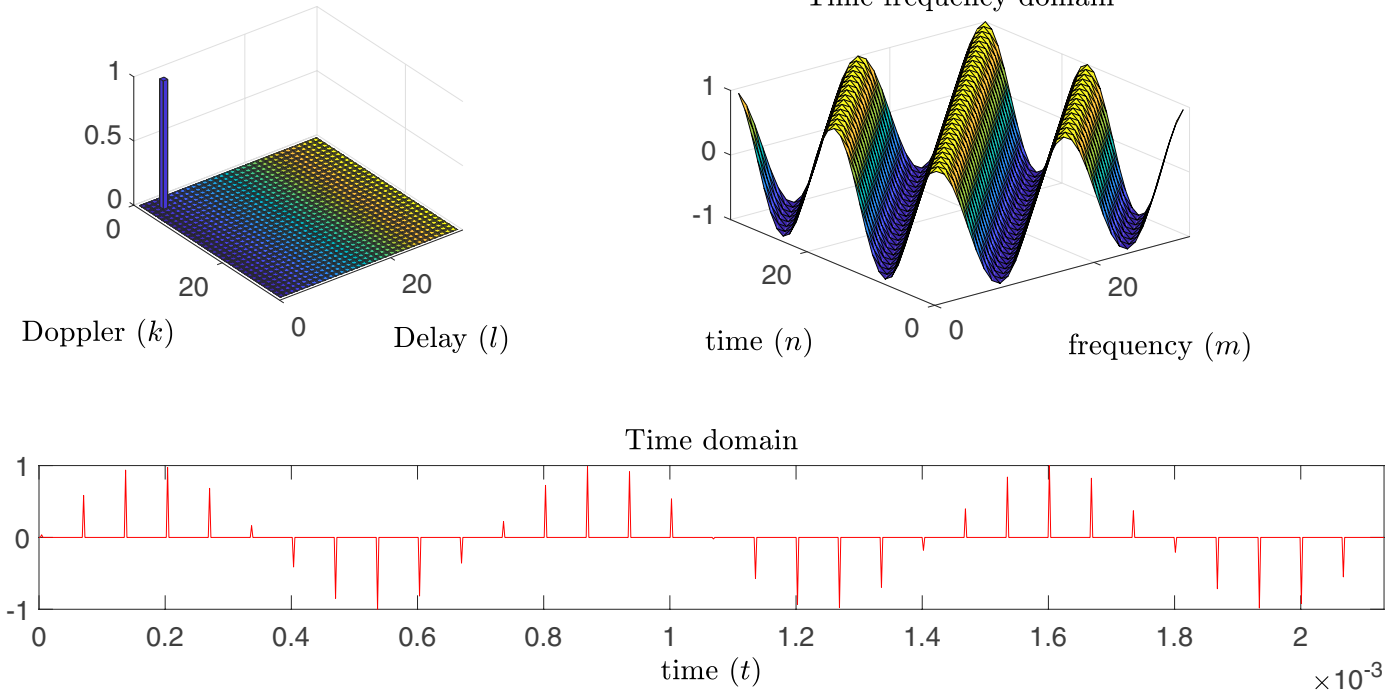

Figure 6: OTFS basis functions in delay-Doppler domain, time-frequency domain, and time domain for Doppler index $k=2$ and delay index $/=2$.

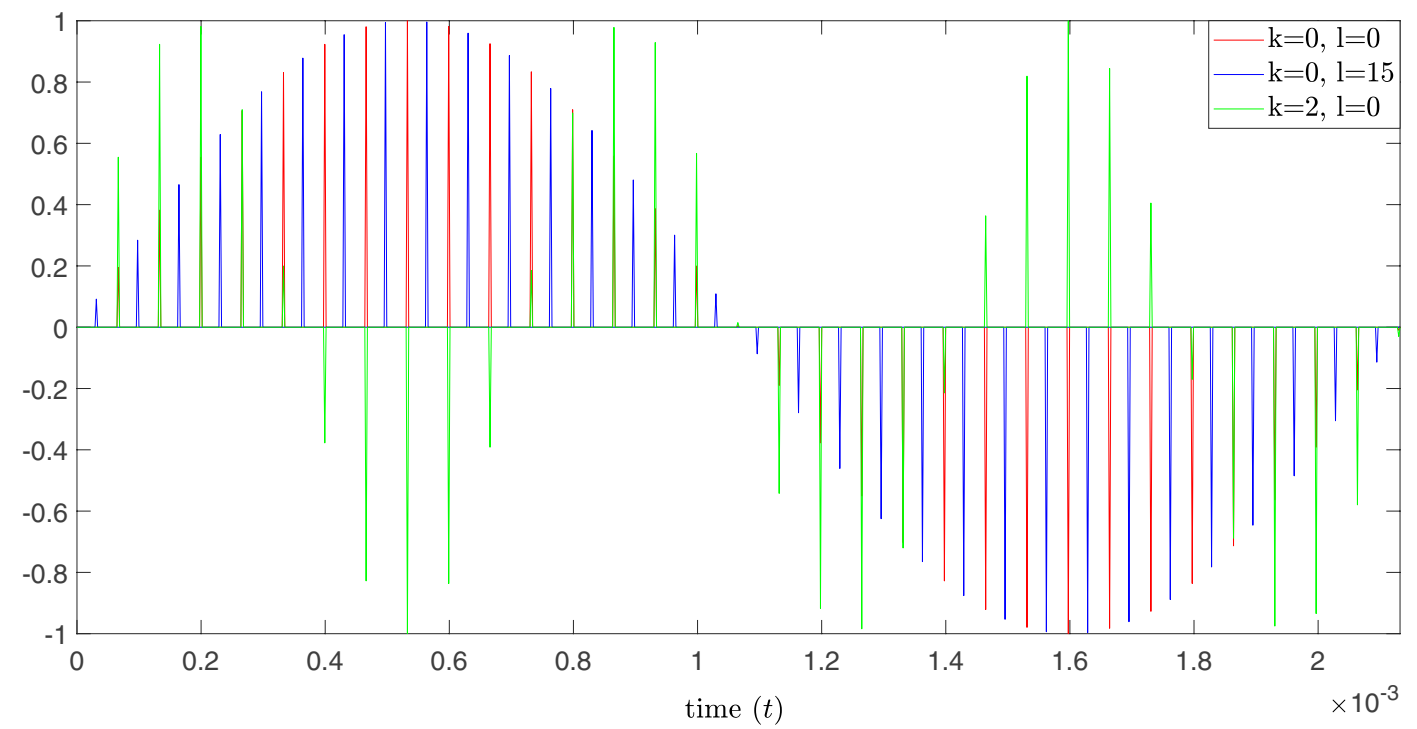

Figure 7: OTFS basis functions in time domain for $(k, l)=(0,0),(k, l)=(0,15)$, and $(k, l)=(2,0)$.

Now, the input-output relation in $(20)$ can be vectorized as ${ }^{18}$

$$
\mathbf{y}=\mathbf{H x}+\mathbf{v}
$$

where

$y_{k+N l}=y[k, l], \quad v_{k+N l}=v[k, l]$, $k=0, \ldots, N-1, l=0, \ldots, M-1, \quad$ and $\mathbf{H} \in \mathbb{C}^{M N \times M N}$, whose $j$ th row $(j=k+N l$, $j=0,1, \ldots, M N-1)$, denoted by $\mathbf{H}[j]$, is given by

$$
\begin{aligned}
\mathbf{H}[j]= & {\left[\hat{h}\left((k-0)_{N},(l-0)_{M}\right)\right.} \\
& \hat{h}\left((k-1)_{N},(l-0)_{M}\right) \\
& \left.\ldots \hat{h}\left((k-N-1)_{N},(l-M-1)_{M}\right)\right] .
\end{aligned}
$$

Note that, due to the modulo operations in (20), each row of $\mathbf{H}$ in (23) has only $P$ non-zero elements. 


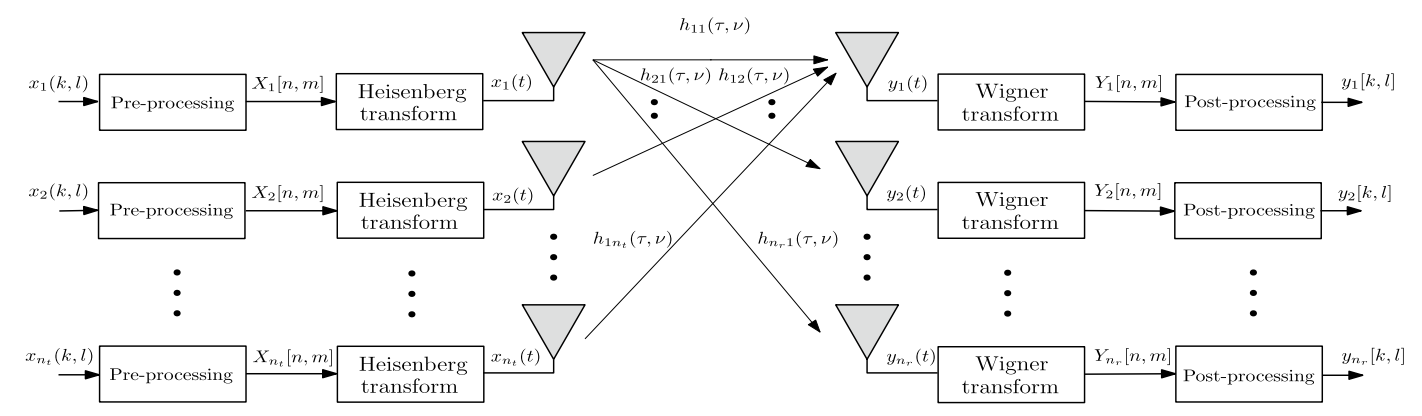

Figure 8: MIMO-OTFS modulation scheme.

\section{MIMO-OTFS Modulation}

Consider a MIMO-OTFS system ${ }^{26}$ with $n_{t}$ transmit antennas and $n_{r}$ receive antennas, $n_{t} \geq n_{r}$ as shown in Fig. 8. Information symbols are OTFS modulated and sent from each of the $n_{t}$ transmit antennas independently. Let the transmit window $W_{t x}[n, m]$ and receive window $W_{r x}[n, m]$ used in OTFS pre- and post-processing be rectangular. The channel between $p$ th transmit and $q$ th receive antennae is assumed to have $P$ taps as in (19). Therefore, the equivalent delay-Doppler channel model is given by

$h_{q p}(\tau, v)=\sum_{i=1}^{P} h_{q p_{i}} \delta\left(\tau-\tau_{i}\right) \delta\left(v-v_{i}\right)$,

$p=1,2, \ldots, n_{t}, q=1,2, \ldots, n_{r}$. Thus, vectorized formulation in Sect. 3.3 for SISO-OTFS can be extended to MIMO-OTFS systems.

\subsection{Vectorized Formulation of the Input- Output Relation for MIMO-OTFS}

Let the channel matrix between the $p$ th transmit antenna and $q$ th receive antenna be denoted by $\mathbf{H}_{q p}$. Let $\mathbf{x}_{p}$ be the vector transmitted from the $p$ th transmit antenna and $\mathbf{y}_{q}$ be the received vector at the $q$ th receive antenna in an OTFS frame. Both are of size $N M \times 1$. Then we derive a linear vectorized input-output model for MIMOOTFS, as described below:

$$
\begin{array}{cc}
\mathbf{y}_{1} & =\mathbf{H}_{11} \mathbf{x}_{1}+\mathbf{H}_{12} \mathbf{x}_{2}+\cdots+\mathbf{H}_{1 n_{t}} \mathbf{x}_{n_{t}}+\mathbf{v}_{1}, \\
\mathbf{y}_{2} & =\mathbf{H}_{21} \mathbf{x}_{1}+\mathbf{H}_{22} \mathbf{x}_{2}+\cdots+\mathbf{H}_{2 n_{t}} \mathbf{x}_{n_{t}}+\mathbf{v}_{2}, \\
\vdots & \vdots \\
\mathbf{y}_{n_{r}} & =\mathbf{H}_{n_{r} 1} \mathbf{x}_{1}+\mathbf{H}_{n_{r} 2} \mathbf{x}_{2}+\cdots+\mathbf{H}_{n_{r} n_{t}} \mathbf{x}_{n_{t}}+\mathbf{v}_{n_{r}} .
\end{array}
$$

Define

$$
\begin{aligned}
\mathbf{H}_{\mathrm{MIMO}} & =\left[\begin{array}{cccc}
\mathbf{H}_{11} & \mathbf{H}_{12} & \ldots & \mathbf{H}_{1 n_{t}} \\
\mathbf{H}_{21} & \mathbf{H}_{22} & \ldots & \mathbf{H}_{2 n_{t}} \\
\vdots & \vdots & \ddots & \vdots \\
\mathbf{H}_{n_{r} 1} & \mathbf{H}_{n_{r} 2} & \ldots & \mathbf{H}_{n_{r} n_{t}}
\end{array}\right] \\
\mathbf{x}_{\mathrm{MIMO}} & =\left[\mathbf{x}_{1}^{T}, \mathbf{x}_{2}^{T}, \ldots, \mathbf{x}_{n_{t}}{ }^{T}\right]^{T} \\
\mathbf{y}_{\mathrm{MIMO}} & =\left[\mathbf{y}_{1}^{T}, \mathbf{y}_{2}{ }^{T}, \ldots, \mathbf{y}_{n_{r}}{ }^{T}\right]^{T} \\
\mathbf{v}_{\mathrm{MIMO}} & =\left[\mathbf{v}_{1}^{T}, \mathbf{v}_{2}{ }^{T}, \ldots, \mathbf{v}_{n_{r}}{ }^{T}\right]^{T}
\end{aligned}
$$

Then (25) can be written as

$$
\mathbf{y}_{\mathrm{MIMO}}=\mathbf{H}_{\mathrm{MIMO}} \mathbf{x}_{\mathrm{MIMO}}+\mathbf{v}_{\mathrm{MIMO}}
$$

where $\mathbf{x}_{\mathrm{MIMO}} \in \mathbb{C}^{n_{t} N M \times 1}, \mathbf{y}_{\mathrm{MIMO}}, \mathbf{v}_{\mathrm{MIMO}} \in \mathbb{C}^{n_{r} N M \times 1}$, $\mathbf{H}_{\text {MIMO }} \in \mathbb{C}^{n_{r} N M \times n_{t} N M}$. Thus due to modulo operations, each row of the matrix $\mathbf{H}_{\mathrm{MIMO}}$ in this representation, has only $n_{t} P$ non-zero elements and each column has only $n_{r} P$ non-zero elements.

\section{Signal Detection for MIMO-OTFS}

We now present an iterative message passing algorithm for MIMO-OTFS signal detection and present results for MIMO-OTFS and MIMO-OFDM performance comparison in highly time-selective multipath fading channels.

\subsection{Signal Detection Algorithm for MIMO-OTFS}

Denote the set consisting of non-zero positions in the $b$ th row and $a$ th column of $\mathbf{H}_{\text {MIMO }}$ by $\zeta_{b}$ and $\zeta_{a}$, respectively. Thus, (26) enables us to model the system as a sparse factor graph having $n_{t} N M$ variable nodes (elements of $\mathbf{x}_{\mathrm{MIMO}}$ ) and $n_{r} N M$ observation nodes (elements of $\mathbf{y}_{\text {MIMO }}$ ). Each observation node $y_{b}$ in the graph is connected to the set of variable nodes $\left\{x_{c}, c \in \zeta_{b}\right\}$, and each variable node $x_{a}$ in the graph is connected to the set of observation nodes $\left\{y_{c}, c \in \zeta_{a}\right\}$. Thus, 
$\left|\zeta_{b}\right|=n_{t} P$ and $\left|\zeta_{a}\right|=n_{r} P$. For the detection of $\mathbf{x}_{\text {MIMO in (26), the maximum a posteriori (MAP) }}$ decision rule is

$\hat{\mathbf{x}}_{\mathrm{MIMO}}=\underset{\mathbf{x}_{\mathrm{MIMO}} \in \mathbb{A}^{n_{t}} t^{N M}}{\operatorname{argmax}} \operatorname{Pr}\left(\mathbf{x}_{\mathrm{MIMO}} \mid \mathbf{y}_{\mathrm{MIMO}}, \mathbf{H}_{\mathrm{MIMO}}\right)$,

where $\mathbb{A}$ is the modulation alphabet (e.g., QAM) used. The exponential complexity of (27) makes it infeasible in practice and can't be used. Instead, we can use a reduced complexity symbol by symbol MAP rule for $0 \leq a \leq n_{t} N M-1$ for detection as given below:

$$
\begin{aligned}
\hat{x}_{a} & =\underset{a_{j} \in \mathbb{A}}{\operatorname{argmax}} \operatorname{Pr}\left(x_{a}=a_{j} \mid \mathbf{y}_{\mathrm{MIMO}}, \mathbf{H}_{\mathrm{MIMO}}\right) \\
& =\underset{a_{j} \in \mathbb{A}}{\operatorname{argmax}} \frac{1}{|\mathbb{A}|} \operatorname{Pr}\left(\mathbf{y}_{\mathrm{MIMO}} \mid x_{a}=a_{j}, \mathbf{H}_{\mathrm{MIMO}}\right) \\
& \approx \underset{a_{j} \in \mathbb{A}}{\operatorname{argmax}} \prod_{c \in \zeta_{a}} \operatorname{Pr}\left(y_{c} \mid x_{a}=a_{j}, \mathbf{H}_{\mathrm{MIMO}}\right) .
\end{aligned}
$$

The elements of $\mathbf{x}_{\text {MIMO }}$ has uniform probability of occurrence and the elements of yMIMO are assumed to be nearly independent for a given $x_{a}$. This assumption is true because of the sparsity of $\mathbf{H}_{\mathrm{MIMO}}$. Iterative message passing based algorithm can be used to solve this problem as described below. The message that is passed from the variable node $x_{a}$, for each $a=\left\{0,1, \ldots, n_{t} N M-1\right\}$, to the observation node $y_{b}$ for $b \in \zeta_{a}$, is the probability mass function (pmf) denoted by $\mathbf{p}_{a b}=\left\{p_{a b}\left(a_{j}\right) \mid a_{j} \in \mathbb{A}\right\}$ of the symbols in the constellation $\mathbb{A}$. Denote the element in the ath row and $b$ th column of $\mathbf{H}_{\text {MIMO }}$ by $H_{a b}$. The message passing algorithm is described below.

1. Inputs $\mathbf{y}_{\mathrm{MIMO}}, \mathbf{H}_{\mathrm{MIMO}}, N_{\text {iter }}$ : max. number of iterations.

2. Initialization Iteration index $t=0, \mathrm{pmf}$ $\mathbf{p}_{a b}^{(0)}=1 /|\mathbb{A}| \forall a \in\left\{0,1, \ldots, n_{t} N M-1\right\}$ and $b \in \zeta_{a}$.

3. Messages from $y_{b}$ to $x_{a}$ The mean $\left(\mu_{b a}^{(t)}\right)$ and variance $\left(\left(\sigma_{b a}^{(t)}\right)^{2}\right)$ of the interference term $I_{b a}$ are passed as messages from $y_{b}$ to $x_{a}$. $I_{b a}$ can be approximated as a Gaussian random variable and is given by

$$
I_{b a}=\sum_{c \in \zeta_{b}, c \neq a} x_{c} H_{b, c}+v_{b} .
$$

The mean and variance of $I_{b a}$ are given by

$$
\begin{aligned}
\mu_{b a}^{(t)}= & \mathbb{E}\left[I_{b a}\right]=\sum_{c \in \zeta_{b}, c \neq a} \sum_{j=1}^{|\mathbb{A}|} p_{c b}^{(t)}\left(a_{j}\right) a_{j} H_{b, c}, \\
\left(\sigma_{b a}^{(t)}\right)^{2}= & \operatorname{Var}\left[I_{b a}\right]=\sum_{\substack{c \in \zeta_{b} \\
c \neq a}}\left(\sum_{j=1}^{|\mathbb{A}|} p_{c b}^{(t)}\left(a_{j}\right)\left|a_{j}\right|^{2}\left|H_{b, c}\right|^{2}\right. \\
& \left.-\left|\sum_{j=1}^{|\mathbb{A}|} p_{c b}^{(t)}\left(a_{j}\right) a_{j} H_{b, c}\right|^{2}\right)+\sigma^{2} .
\end{aligned}
$$

4. Messages from $x_{a}$ to $y_{b}$ Messages passed from variable nodes $x_{a}$ to observation nodes $y_{b}$ is the pmf vector $\mathbf{p}_{a b}^{(t+1)}$ with the elements given by

$p_{a b}^{(t+1)}=\Delta p_{a b}^{(t)}\left(a_{j}\right)+(1-\Delta) p_{a b}^{(t-1)}\left(a_{j}\right)$,

where $\Delta \in(0,1]$ is the damping factor for improving convergence rate, and

$p_{a b}^{(t)} \propto \prod_{c \in \zeta_{a}, c \neq b} \operatorname{Pr}\left(y_{c} \mid x_{a}=a_{j}, \mathbf{H}_{\mathrm{MIMO}}\right)$

where

$$
\begin{aligned}
& \operatorname{Pr}\left(y_{c} \mid x_{a}=a_{j}, \mathbf{H}_{\mathrm{MIMO}}\right) \\
& \quad \propto \exp \left(\frac{-\left|y_{c}-\mu_{c a}^{(t)}-H_{c, a} a_{j}\right|^{2}}{\sigma_{c, a}^{2(t)}}\right) .
\end{aligned}
$$

5. Stopping criterion Repeat steps $3 \& 4$ till $\max _{a, b, a_{j}}\left|p_{a b}^{(t+1)}\left(a_{j}\right)-p_{a b}^{(t)}\left(a_{j}\right)\right|<\epsilon$ (where $\epsilon$ is a small value) or the maximum number of iterations, $N_{i t e r}$, is reached.

6. Output Output the detected symbol as

$$
\hat{x}_{a}=\underset{a_{j} \in \mathbb{A}}{\operatorname{argmax}} p_{a}\left(a_{j}\right), a \in 0,1,2, \ldots, n_{t} N M-1,
$$

where

$$
p_{a}\left(a_{j}\right)=\prod_{c \in \zeta_{a}} \operatorname{Pr}\left(y_{c} \mid x_{a}=a_{j}, \mathbf{H}_{\mathrm{MIMO}}\right) .
$$

\subsection{Vectorized Formulation of the Input- Output Relation for MIMO-OFDM}

Performance comparison of MIMO-OTFS and MIMO-OFDM can be done by first formulating an input-output vector relation for MIMO-OFDM similar to MIMO-OTFS. In this section, we develop this formulation in time-frequency domain, which is the signaling domain for MIMO-OFDM. Vectorized formulation of 
MIMO-OFDM can be easily obtained by extending the results of SISO-OFDM as shown below. Consider $N$ consecutive blocks (each of size $M$ ) to be one OFDM frame, i.e., the transmit vector $\mathbf{x}_{\text {OFDM }} \in \mathbb{C}^{N M \times 1}$ (This is for a fair comparison with OTFS in terms of spectral efficiency). This means, iterative message passing algorithm is jointly applied to the $N M \times 1$ frame. Consider the channel in (19). The time-delay representation $h(\tau, t)$ and the delay-Doppler representation $h(\tau, v)$ are related by a Fourier transform along the time axis, and is given by

$h(\tau, t)=\sum_{i=1}^{P} h_{i} e^{j 2 \pi v_{i} t} \delta\left(\tau-\tau_{i}\right)$.

Sample the time axis at $t=n T s=\frac{n}{M \Delta f}$. The sampled time-delay representation $h(\tau, n)$ is given by

$h(\tau, n)=\sum_{i=1}^{P} h_{i} e^{\frac{j 2 \pi v_{i} n}{M \Delta f}} \delta\left(\tau-\tau_{i}\right)$.

In each OFDM block, let $C P=P-1$ be the cyclic prefix (CP) length used and also let $L=M+C P$. Thus, the size of one OFDM frame after CP insertion to each block is $N L$. Let $\mathbf{T}_{C P}=\left[\begin{array}{ll}\mathbf{C}_{C P}^{T} & \mathbf{I}_{M}\end{array}\right]^{T}$ denote the $L \times M \mathrm{CP}$ insertion matrix per block, where $\mathbf{C}_{C P}$ consists of the last $C P$ rows of the identity matrix $\mathbf{I}_{M}$. Also, let $\mathbf{R}_{C P}=\left[\mathbf{0}_{M \times C P} \mathbf{I}_{M}\right]$ be the $M \times L$ matrix which does the cyclic prefix removal for each block ${ }^{41}$. Denote the DFT and
IDFT matrices of size $M$ by $\mathbf{W}_{M \times M}$ and $\mathbf{W}_{M \times M}^{H}$, respectively. The following notations are used:

- $\mathbf{B}_{\text {cpin }}=\left(\mathbf{I}_{N} \otimes \mathbf{T}_{C P}\right)$ : cyclic prefix insertion matrix for $N$ consecutive OFDM blocks.

- $\mathbf{B}_{\text {cpre }}=\left(\mathbf{I}_{N} \otimes \mathbf{R}_{C P}\right)$ : cyclic prefix removal matrix for $N$ consecutive OFDM blocks.

- $\mathbf{D}=\left(\mathbf{I}_{N} \otimes \mathbf{W}\right)$ : DFT matrix for $N$ consecutive OFDM blocks.

- $\mathbf{D}^{H}=\left(\mathbf{I}_{N} \otimes \mathbf{W}^{H}\right)$ : IDFT matrix for $N$ consecutive OFDM blocks.

- The time-delay domain channel for a given OFDM frame can be written as a matrix $\mathbf{H}_{t d}$ of size $N L \times N L$, using (35).

Thus, the linear input-output vector model for SISO-OFDM is given by

$$
\begin{aligned}
\mathbf{y}_{\text {OFDM }} & =\underbrace{\mathbf{D B}_{c p r e} \mathbf{H}_{t d} \mathbf{B}_{c p i n} \mathbf{D}^{H}}_{\mathbf{H}_{\text {OFDM }}} \mathbf{x}_{\text {OFDM }}+\mathbf{v} \\
& =\mathbf{H}_{\text {OFDM }} \mathbf{x}_{\text {OFDM }}+\mathbf{v},
\end{aligned}
$$

where $\quad \mathbf{x}_{\text {OFDM }}$, YOFDM, $\mathbf{v} \in \mathbb{C}^{N M \times 1}$, $\mathbf{H}_{\text {OFDM }} \in \mathbb{C}^{N M \times N M}$.

\subsubsection{MIMO-OFDM}

In this subsection, we extend the SISO-OFDM results derived above to MIMO-OFDM. Denote the equivalent channel matrix between $p$ th transmit antenna and $q$ th receive antenna by $\mathbf{H}_{\mathrm{OFDM}_{q p}}$. Let $\mathbf{x}_{\mathrm{OFDM}_{p}}$ of size $N M \times 1$ be the transmit vector from the $p$ th transmit antenna and yofDM $_{q}$ of size $N M \times 1$ be the received vector at the $q$ th receive antenna in a given OFDM frame. Define

$\mathbf{H}_{\mathrm{MIMO}-\mathrm{OFDM}}=\left[\begin{array}{cccc}\mathbf{H}_{\mathrm{OFDM}_{11}} & \mathbf{H}_{\mathrm{OFDM}_{12}} & \ldots & \mathbf{H}_{\mathrm{OFDM}_{1 n_{t}}} \\ \mathbf{H}_{\mathrm{OFDM}_{21}} & \mathbf{H}_{\mathrm{OFDM}_{22}} & \ldots & \mathbf{H}_{\mathrm{OFDM}_{2 n_{t}}} \\ \vdots & \vdots & \ddots & \vdots \\ \mathbf{H}_{\mathrm{OFDM}_{n_{r} 1}} & \mathbf{H}_{\mathrm{OFDM}_{n_{r} 2}} & \ldots & \mathbf{H}_{\mathrm{OFDM}_{n_{r} n_{t}}}\end{array}\right]$

and

$\mathbf{x}_{\text {MIMO-OFDM }}=\left[\mathbf{x}_{\mathrm{OFDM}_{1}}{ }^{T}, \mathbf{x}_{\mathrm{OFDM}_{2}}{ }^{T}, \cdots, \mathbf{x}_{\mathrm{OFDM}_{n_{t}}}{ }^{T}\right]^{T}$,

$\mathbf{y}_{\text {MIMO-OFDM }}=\left[\mathbf{y O F D M}_{1}{ }^{T}, \text { yOFDM }_{2}{ }^{T}, \cdots, \text { yOFDM }_{n_{r}}{ }^{T}\right]^{T}$. 


Table 1: Delay-Doppler profile for the channel
model with $\boldsymbol{P}=\mathbf{5}$ at $4 \mathrm{GHz}$ carrier frequencv.
\begin{tabular}{lllllll}
\hline Path index $(i)$ & 1 & 2 & 3 & 4 & 5 \\
\hline Delay $\left(\tau_{i}\right)(\mu \mathrm{s})$ & 2.08 & 4.164 & 6.246 & 8.328 & 10.41 \\
Doppler $\left(v_{i}\right)(\mathrm{Hz})$ & 0 & 470 & 940 & 1410 & 1880 \\
Speed $(\mathrm{km} / \mathrm{h})$ & 0 & 126.9 & 253.8 & 380.7 & 507.6 \\
\hline
\end{tabular}

\begin{tabular}{ll} 
Table 2: Svstem parameters for 4 GHz svstem. \\
\hline Parameter & Value \\
\hline Carrier frequency $(\mathrm{GHz})$ & 4 \\
Frame size $(M, N)$ & $(32,32)$ \\
Subcarrier spacing $(\mathrm{kHz})$ & 15 \\
Modulation scheme & BPSK \\
MIMO configuration & $1 \times 1,1 \times 2$, \\
& $1 \times 3,2 \times 3$, \\
& $2 \times 2,3 \times 3$ \\
No. of taps, $P$ & 5 \\
Maximum speed $(\mathrm{km} / \mathrm{h})$ & 507.6 \\
\hline
\end{tabular}

Thus, the linear vectorized input-output model for MIMO-OFDM is given by

$$
\begin{aligned}
\mathbf{y}_{\text {MIMO-OFDM }}= & \mathbf{H}_{\text {MIMO-OFDM }} \mathbf{x}_{\text {MIMO-OFDM }} \\
& +\mathbf{v}_{\text {MIMO }}
\end{aligned}
$$

where $\mathbf{x}_{\text {MIMO-OFDM }} \in \mathbb{C}^{n_{t} N M \times 1}, \mathbf{y}_{\text {MIMO-OFDM }}$, $\mathbf{v}_{\text {MIMO }} \in \mathbb{C}^{n_{r} N M \times 1}$

$\mathbf{H}_{\mathrm{MIMO}-\mathrm{OFDM}} \in \mathbb{C}^{n_{r} N M \times n_{t} N M}$.

\subsection{Performance Results and Discussion}

In this subsection, the BER performance comparison of MIMO-OTFS and MIMO-OFDM in high-mobility channel scenarios, is presented. The channel knowledge at the receiver is assumed to be perfect. Iterative message passing-based detection is used for both MIMO-OTFS and MIMO-OFDM. The algorithm is run for 30 iterations with an $\epsilon$ value 0.01 . The channel model in (24) is used with the number of taps $P=5$. For all the paths the fade coefficients $h_{i}$ are assumed to be distributed as $\mathcal{C N}(0,1)$, i.e., Rayleigh fading. The channel fade coefficients are simulated as per (21) with the delay-Doppler profile $\left\{\left(\tau_{i}, v_{i}\right), i=1, \ldots, P\right\}$ shown in Table 1 . Table 2 gives all other simulation parameters which were used in the simulations. The carrier frequency and subcarrier spacing used are $4 \mathrm{GHz}$ and 15 $\mathrm{kHz}$, respectively, the frame size parameters used are $M=N=32$, and the modulation used is BPSK.

Figure 9 shows the BER performance of OTFS in SISO $(1 \times 1)$ and SIMO $(1 \times 2$ and $1 \times 3)$ settings, Fig. 10 shows the performance in $2 \times 2$ and $2 \times 3 \mathrm{MIMO}$ settings, and Fig. 11 shows the performance in SISO and $2 \times 2,3 \times 3$ MIMO settings. The maximum considered Doppler of $1880 \mathrm{~Hz}$ corresponds to a speed of $507.6 \mathrm{~km} / \mathrm{h}$ at $4 \mathrm{GHz}$ carrier frequency. This means that, the channel is highly time-selective. But, from the results we see that MIMO-OTFS BER performance is good, even in such scenarios. For example, we see in Fig. 9 that, at an SNR of about 8 $\mathrm{dB}$, the $1 \times 2$ system achieves a BER of about $10^{-4}$ and the $1 \times 3$ system achieves a BER of

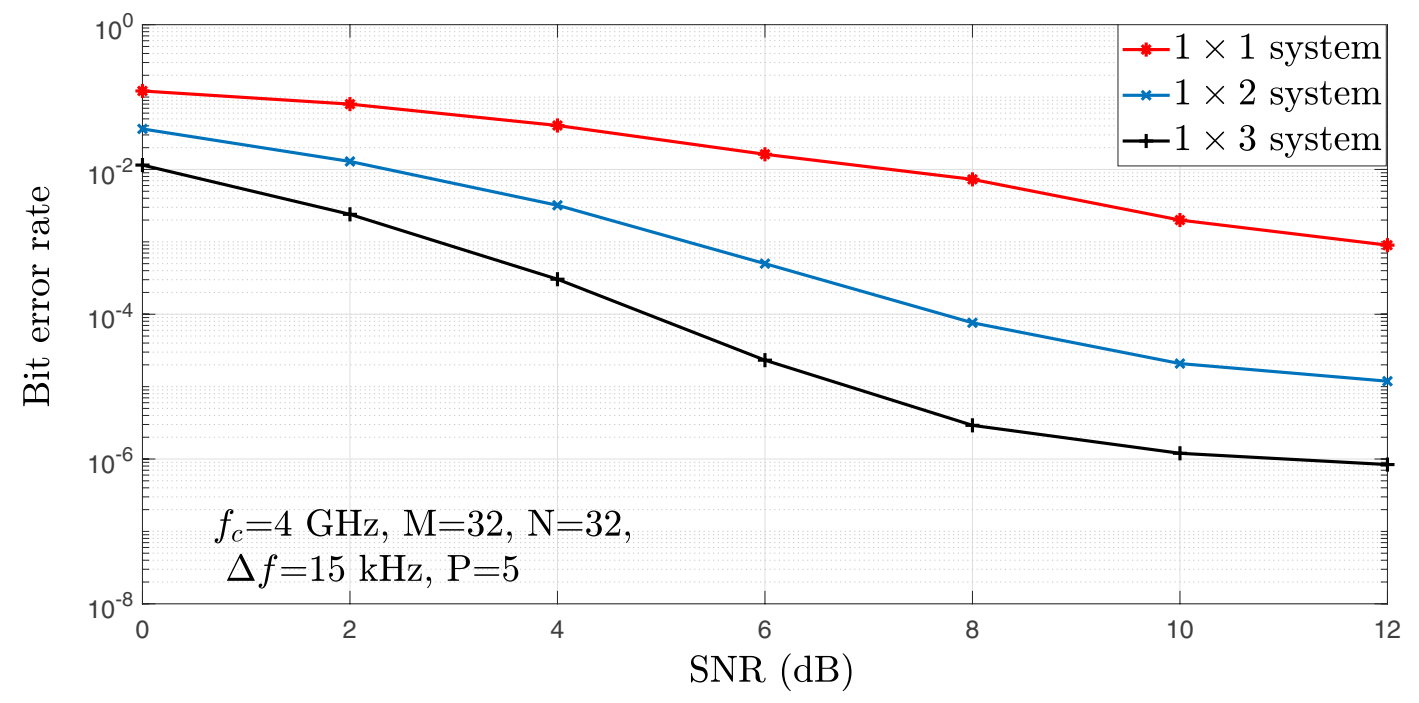

Figure 9: BER performance of OTFS in SISO $(1 \times 1)$ and SIMO $(1 \times 2$ and $1 \times 3)$ settings. 


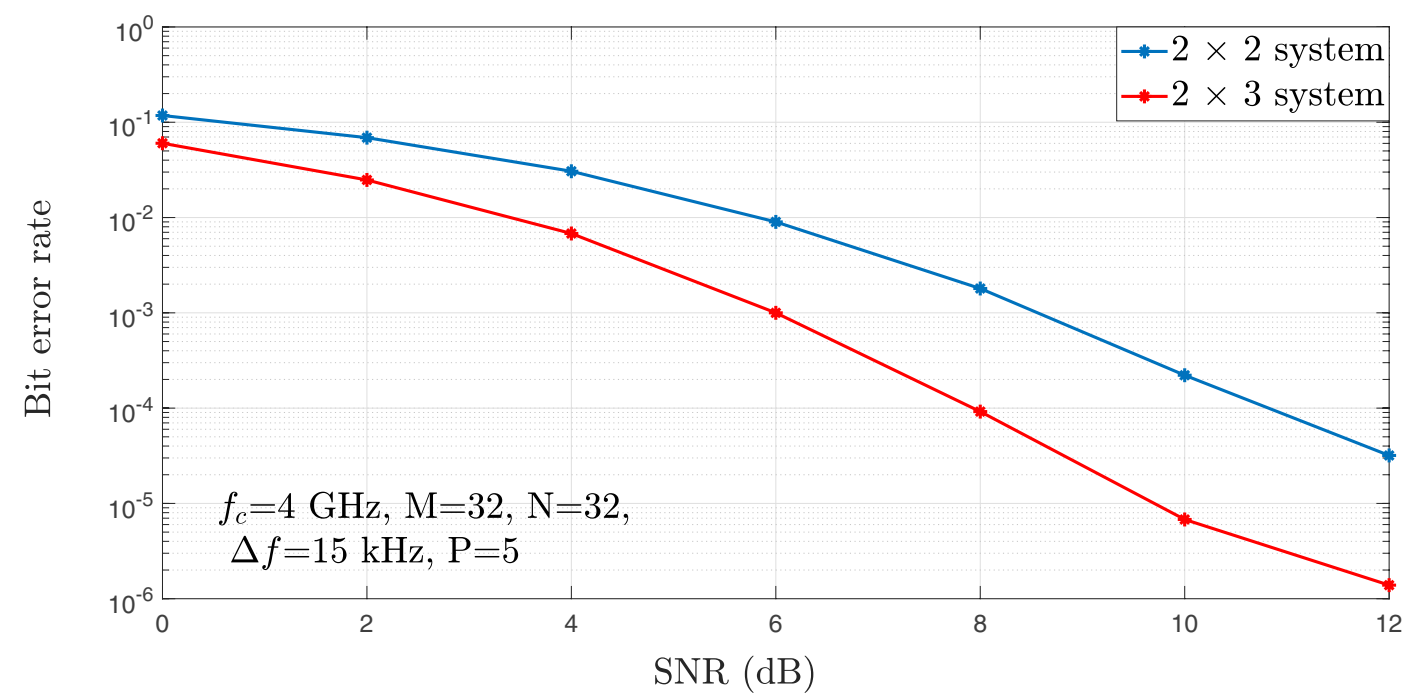

Figure 10: BER performance of MIMO-OTFS in $2 \times 2$ and $2 \times 3$ MIMO systems.

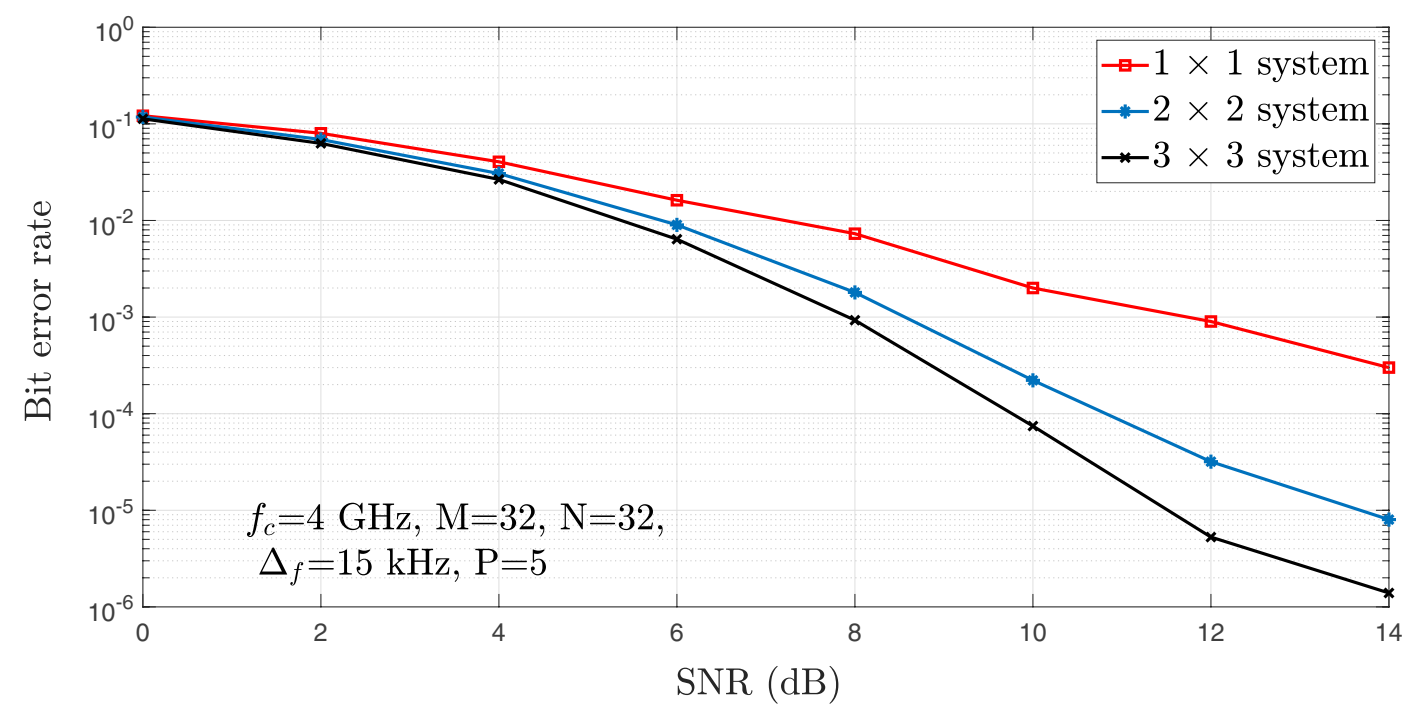

Figure 11: BER performance of MIMO-OTFS in $2 \times 2$ and $3 \times 3$ MIMO systems.

$3 \times 10^{-6}$. Observing that the $1 \times 1$ SISO system achieves a BER of about 0.01 at this SNR, we see that OTFS achieves improved BER performance for increased number of receive antennas even in high Doppler scenarios. This can also be observed in Fig. 10, where a BER of $3 \times 10^{-5}$ is achieved at an SNR of about $12 \mathrm{~dB}$ in a $2 \times 2$ MIMO system, whereas the same BER is achieved at an SNR of about $8.8 \mathrm{~dB}$ in a $2 \times 3 \mathrm{MIMO}$ system. Figure. 11 shows the expected improved performance for increasing $n_{t}=n_{r}$. Thus, with the use of the low-complexity message passing detection, we see that MIMO-OTFS brings in the advantages of linear increase in throughput with number of transmit-receive antennas and the resilience of OTFS modulation to delay-Doppler shifts in doubly selective channels.

Figure 12 shows the BER performance of $2 \times 2$ MIMO-OTFS with (1) message passing detection, (2) minimum mean square error (MMSE) detection, and (3) zero forcing (ZF) detection. The system and channel parameters used are as given in Tables 1 and 2. From Fig. 12, it can be observed that the performance of MIMO-OTFS with message passing detection is superior compared to those of MMSE and ZF detectors. For example, at an SNR of $12 \mathrm{~dB}$, message passing detection achieves a BER of $3 \times 10^{-5}$, whereas MMSE and 


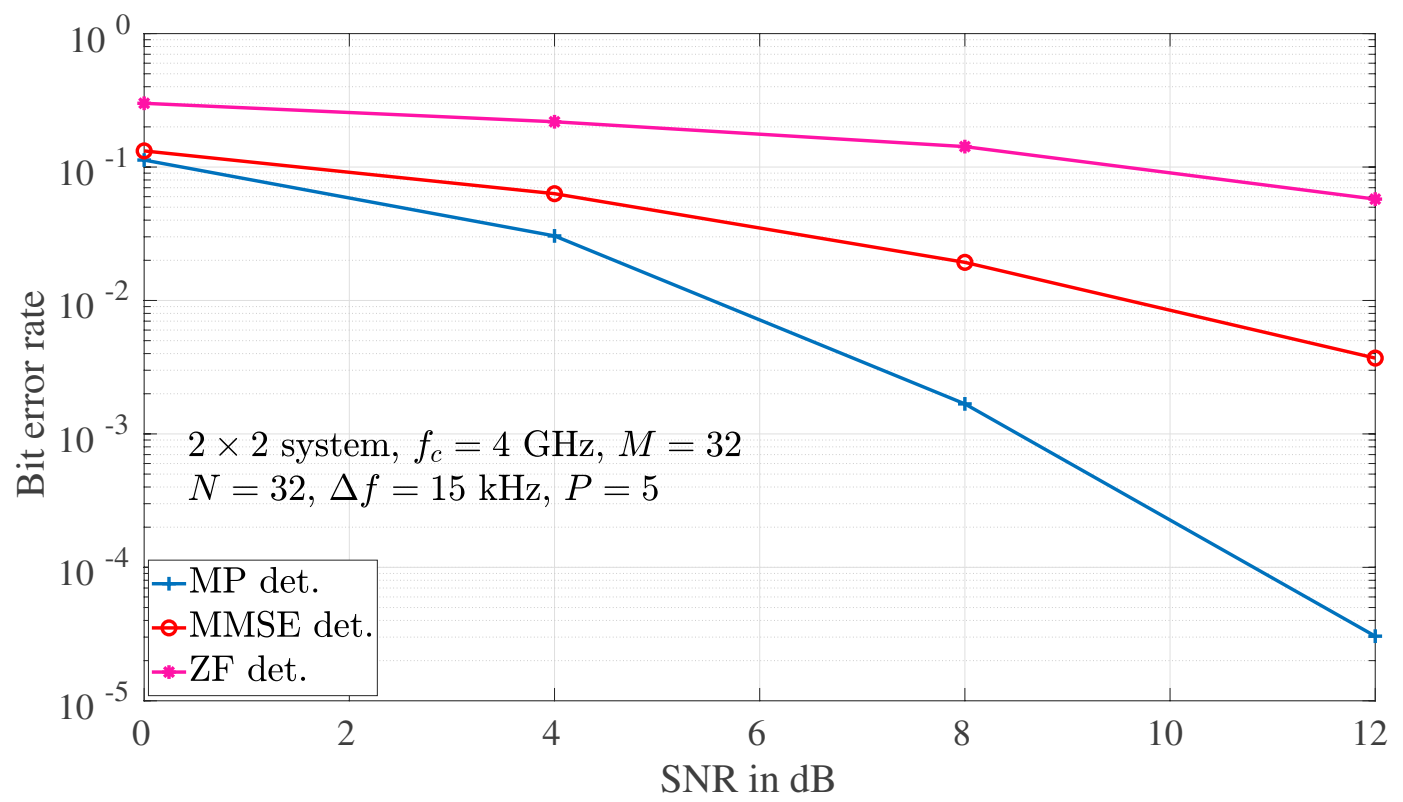

Figure 12: BER performance of $2 \times 2$ MIMO-OTFS system with (1) message passing detection, (2) minimum mean square error (MMSE) detection, and (3) zero forcing (ZF) detection.

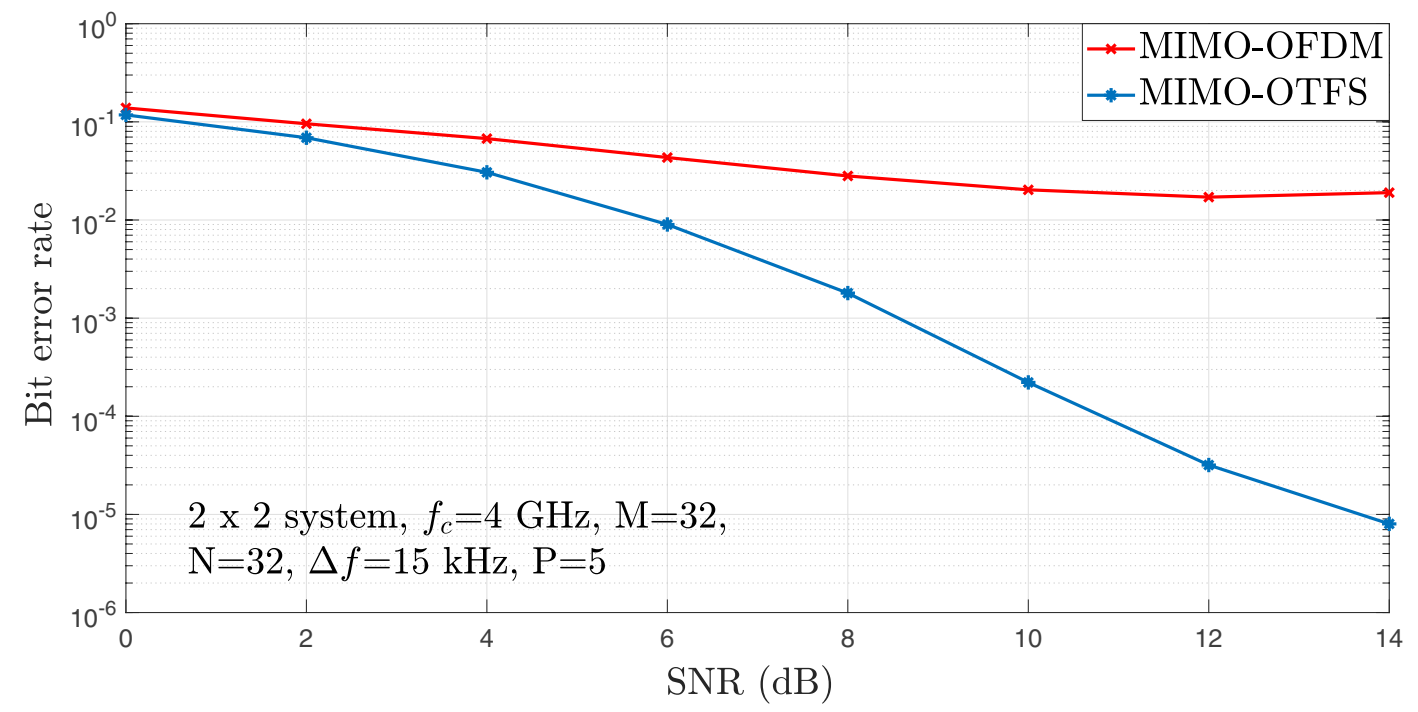

Figure 13: BER performance comparison between MIMO-OTFS and MIMO-OFDM in 2 × 2 MIMO system.

ZF detectors achieve a BER of 0.003 and 0.05 , respectively.

MIMO-OTFS vs MIMO-OFDM Figure 13 shows a comparison of BER performance between MIMO-OTFS and MIMO-OFDM in a $2 \times 2$ MIMO system with the message passingbased detection. For the case of maximum Doppler of $1880 \mathrm{~Hz}$, MIMO-OFDM performance degrades due to inter carrier interference (ICI) in the time-frequency domain. In fact, the BER floors at a value of about $2 \times 10^{-2}$, whereas MIMO-OTFS achieves a BER of $10^{-5}$ at an SNR value close to $14 \mathrm{~dB}$. This illustrates the robust performance of MIMO-OTFS where the signaling is in the delay-Doppler domain and the performance superiority of MIMO-OTFS over MIMO-OFDM under doubly selective channels. 


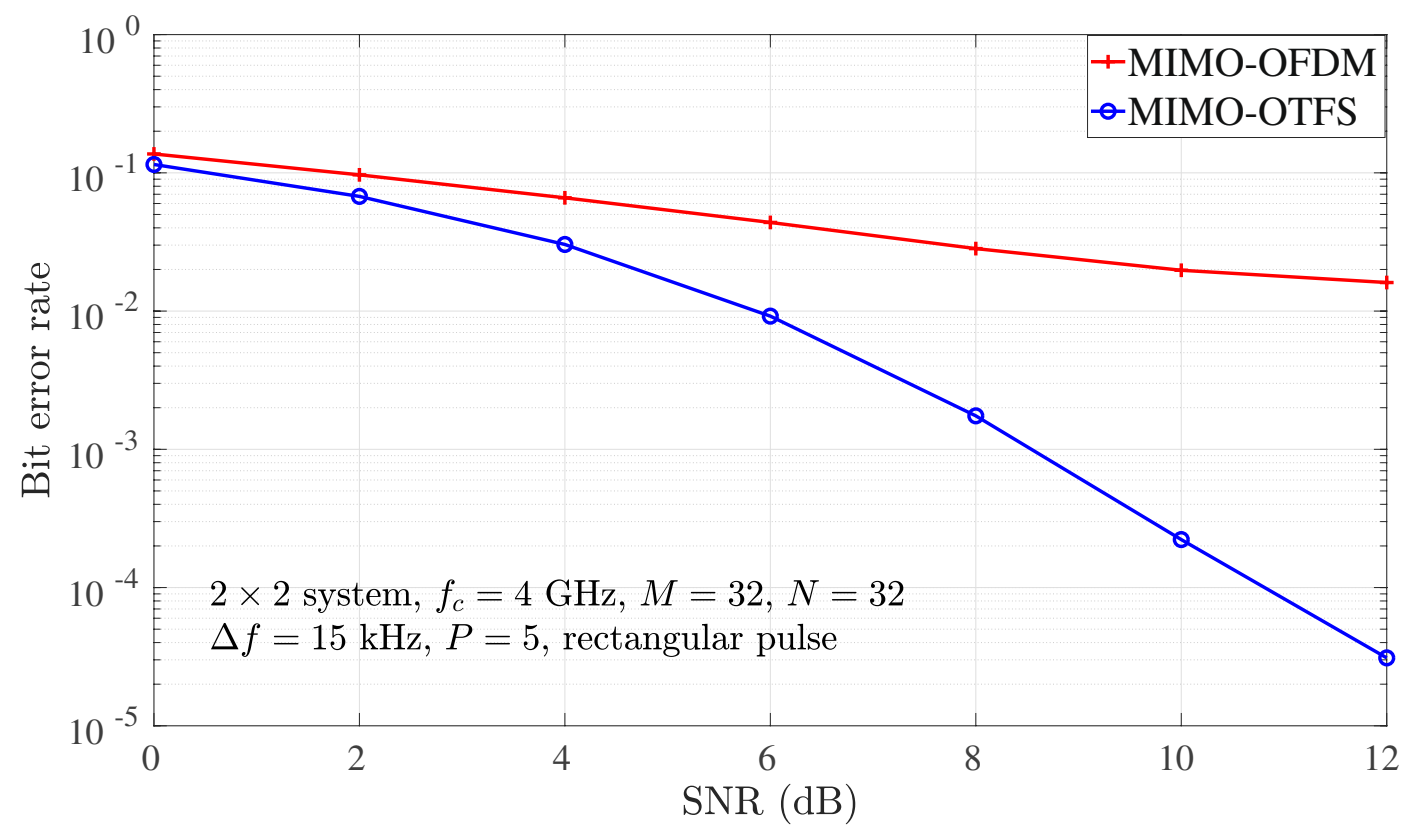

Figure 14: BER performance comparison between $2 \times 2$ MIMO-OTFS with rectangular pulse and $2 \times 2$ MIMO-OFDM.

\subsection{Performance of MIMO-OTFS with Rectangular Pulse}

In this subsection, we consider OTFS modulation with rectangular transmit and receive pulses ${ }^{16,24}$. Figure 14 shows a BER performance comparison between MIMO-OFDM and MIMO-OTFS with rectangular pulse shape in a $2 \times 2$ system. Both the systems use message passing detector for signal detection. The maximum Doppler considered in $1880 \mathrm{kHz}$, which corresponds to a speed of 507.6 $\mathrm{km} / \mathrm{h}$ at a carrier frequency of $4 \mathrm{GHz}$. The delayDoppler profile used for the simulation is given in Table 1. Other parameters used for the simulations are given in Table 2. From the figure, we observe that MIMO-OTFS shows better BER performance compared to MIMO-OFDM even with the use of rectangular pulse. For example, at an SNR of 12 $\mathrm{dB}$, MIMO-OTFS achieves a BER of $3 \times 10^{-5}$, whereas MIMO-OFDM floors at $2 \times 10^{-2}$. This SNR gain shown by OTFS modulation is because of the outer pre- and post-processing operations involving the 2D ISFFT and SFFT that spread the information across the entire time-frequency plane, which enables OTFS to extract higher diversity performance in the finite SNR regime.

\subsection{Application of MIMO-OTFS to mmWave Communications}

In this subsection, we consider mmWave MIMO communications in a point-to-point setting and compare the BER performance of MIMO-OTFS and MIMO-OFDM operating in this setting. We consider a carrier frequency of $28 \mathrm{GHz}$. The transmitter consists of a uniform linear array (ULA) with $n_{t}$ transmit antennas with an interantenna spacing of $d_{t}$. Likewise, the receiver consists of a ULA with $n_{r}$ receive antennas with an inter-antenna spacing of $d_{r}$. As before, the channel model in (24) with $P$ taps is considered. The propagation in typical mmWave frequency application scenarios is dominated by a line-ofsight (LOS) component ${ }^{37}$. Because of this, the magnitude of the first tap in the channel model is considered to follow a Rician distribution and the magnitudes of the remaining taps are considered to follow Rayleigh distribution. Therefore, the fading channel gain in the first tap between the $p$ th transmit antenna and $q$ th receive antenna can be written in the form ${ }^{37}$

$h_{q p_{1}}=\sqrt{\frac{K}{K+1}} h_{q p_{1}}^{\mathrm{LOS}}+\sqrt{\frac{1}{K+1}} h_{q p_{1}}^{\mathrm{NLOS}}$,

where $h_{q p_{1}}^{\mathrm{LOS}}$ and $h_{q p_{1}}^{\mathrm{NLOS}}$ denote the LOS and nonLOS channel gains, respectively, in the first tap between the $p$ th transmit antenna and $q$ th receive antenna, and $K$ is the Rician factor, defined as the ratio of the powers in the LOS and non-LOS components. The LOS gain $h_{q p_{1}}^{\mathrm{LOS}}$ is given by ${ }^{38}$ 


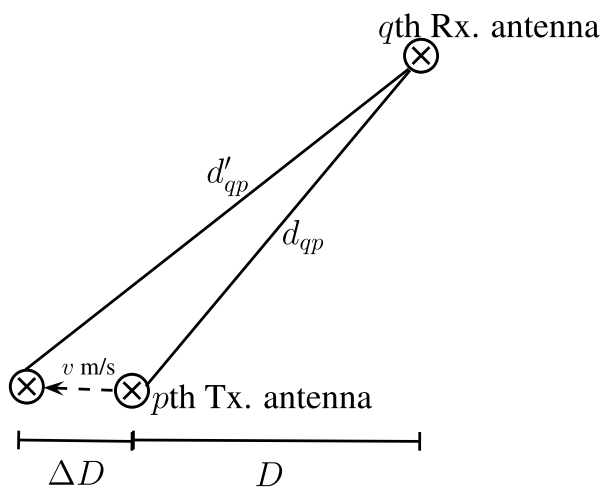

Figure 15: $h_{q p_{1}}^{\mathrm{LOS}}$ in mmWave communication.

$$
h_{q p_{1}}^{\mathrm{LOS}}=e^{-j \frac{2 \pi}{\lambda} d_{q p}},
$$

where $d_{q p}$ denotes the LOS path length between the $p$ th transmit and $q$ th receive antenna, and $\lambda$ is the carrier wavelength.

Figure 15 illustrates the LOS path between the $p$ th transmit antenna and the $q$ th receive antenna when there is relative motion between the transmitter and the receiver. Note that the change in LOS path lengths due to the relative motion between the transmitter and the receiver in one OTFS frame duration is very small for low/medium mobility. This can be illustrated with an example, where we consider $n_{t}=n_{r}=2, d_{t}=d_{r}=5 \mathrm{~cm}$, and $D=90 \mathrm{~m}$.
With $M=N=128, T=12.8 \mu \mathrm{s}$, the duration of one OTFS frame is $N T=1.63 \mathrm{~ms}$. Considering the relative velocity between the transmitter and the receiver to be $v=47 \mathrm{~km} / \mathrm{h}$, the change in the distance between the transmitter and the receiver in one OTFS frame can be calculated as $\Delta D=v N T=0.021 \mathrm{~m}$. For illustration, consider the distance between the fourth transmit antenna and the second receive antenna (i.e., $p=2$, $q=4)$. Let $d_{24}$ and $d_{24}^{\prime}$ denote the LOS path lengths between the fourth transmit and second receive antenna at the beginning and at the end of an OTFS frame, respectively. Now, $d_{24}$ and $d_{24}^{\prime}$ can be calculated to be $90.00005 \mathrm{~m}$ and $90.02 \mathrm{~m}$, respectively. Since the difference between $d_{24}$ and $d_{24}^{\prime}$ is very small, $d_{q p}$ in (39) can be assumed to be constant over one OTFS frame.

We also assume $h_{q p_{1}}^{\mathrm{NLOS}}$ to be i.i.d and distributed as $\mathcal{C N}(0,1)$. Also, the fading gains in the other taps with no LOS component (i.e., Rice factor $K$ in those taps is zero), $h_{q p_{i}}^{\mathrm{NLOS}}$, $i=2, \ldots, P$, are assumed to be i.i.d and distributed as $\mathcal{C N}(0,1)$. The performance is evaluated for SISO and $2 \times 2$ MIMO systems. Table 3 gives the delay-Doppler profile used. Table 4 gives all other parameter values used in simulations. The carrier frequency and subcarrier spacing used are $28 \mathrm{GHz}$ and $78.125 \mathrm{kHz}$, respectively, the frame size parameters used are $M=N=128$, the inter-antenna distances at the transmitter and the receiver are taken as $d_{t}=d_{r}=6.9 \mathrm{~cm}$, and the

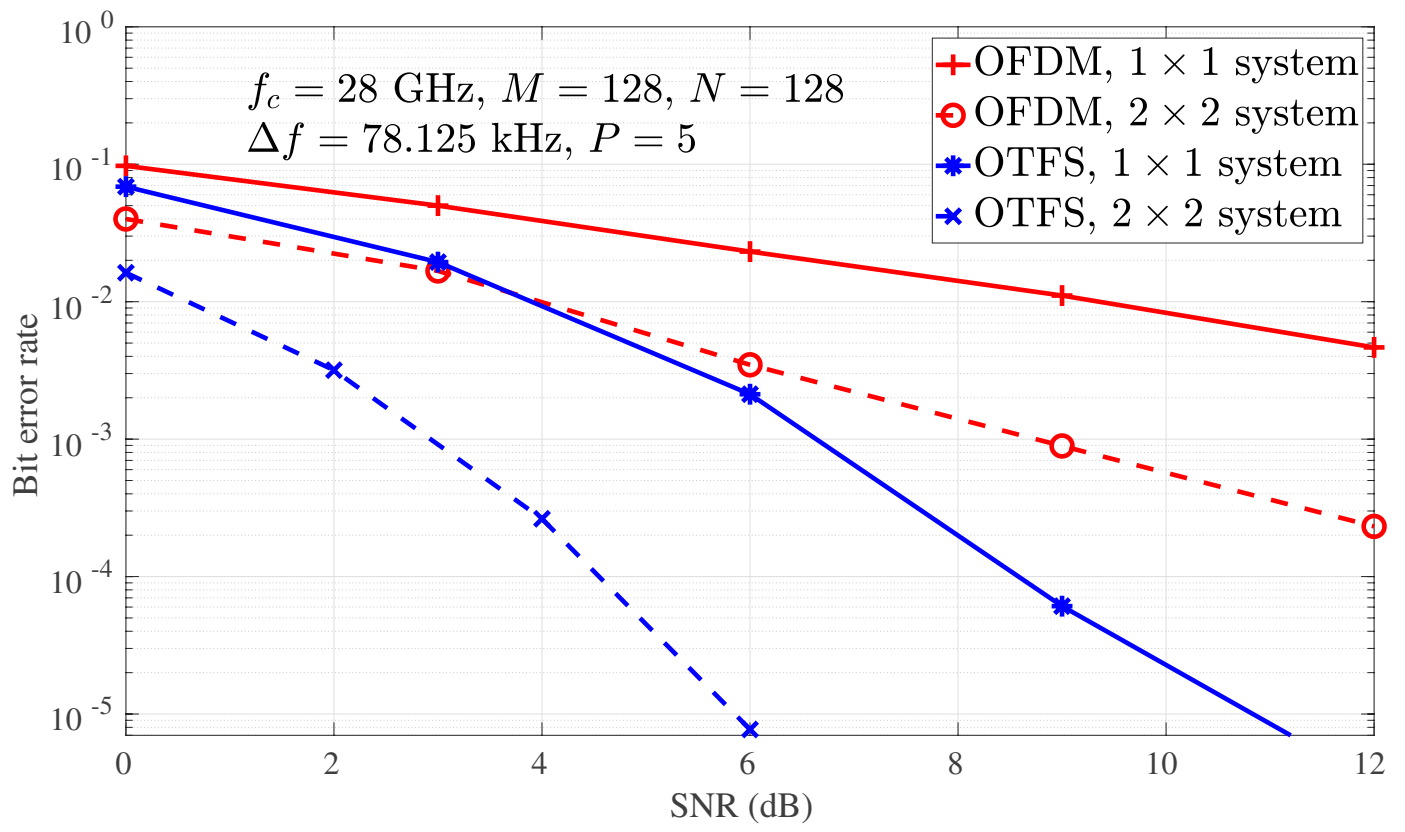

Figure 16: BER performance comparison between MIMO-OTFS and MIMO-OFDM in $2 \times 2$ MIMO system at $28 \mathrm{GHz}$. 


Table 3: delay-Doppler profile for the channel
model with $\boldsymbol{P}=\mathbf{5}$ at $28 \mathrm{GHz}$ carrier freauencv.
\begin{tabular}{lllllll}
\hline Path index $(i)$ & 1 & 2 & 3 & 4 & 5 \\
\hline Delay $\left(\tau_{i}\right)(\mu \mathrm{s})$ & 0.3 & 1 & 1.7 & 2.4 & 3.1 \\
Doppler $\left(v_{i}\right)(\mathrm{Hz})$ & 0 & -1220 & -610 & 610 & 1220 \\
Speed $(\mathrm{km} / \mathrm{h})$ & 0 & 47 & 23.5 & 23.5 & 47 \\
\hline
\end{tabular}

modulation used is BPSK. A Rician factor of 9 $\mathrm{dB}$ as reported in ${ }^{37}$ from $28 \mathrm{GHz}$ band measurements is used.

In Fig. 16, we present a BER performance comparison between OTFS and OFDM in SISO and $2 \times 2 \mathrm{MIMO}$ systems at $28 \mathrm{GHz}$. Iterative message passing is used for both MIMOOTFS and MIMO-OFDM detection. From Fig. 16, it can be observed that SISO-OFDM system achieves a BER of $10^{-2}$ at an SNR of $9 \mathrm{~dB}$, whereas SISO-OTFS achieves a BER of $6 \times 10^{-5}$ for the same SNR. It is also evident from the figure that MIMO-OTFS performs significantly better compared to MIMO-OFDM at $28 \mathrm{GHz}$. For example, it can be seen that $2 \times 2$ MIMO-OTFS system outperforms $2 \times 2$ MIMO-OFDM system by about $8 \mathrm{~dB}$ at a BER of $2 \times 10^{-4}$. This illustrates the robustness of MIMO-OTFS over MIMO-OFDM in high Doppler scenarios that arise due to the use of high carrier frequencies in the mmWave band.

\section{Channel Estimation in delay-Doppler Domain}

In this section, the assumption of availability of perfect channel state information at the receiver (CSIR) is relaxed. We present a simple scheme to estimate the channel in the delay-Doppler domain ${ }^{26}$. In this scheme of channel estimation, impulse function in the delay-Doppler domain is used as the pilot. The pilot corresponding to each antenna is placed in the delay-Doppler grid such they can be received without interference at the receive antennas. Figure 17 illustrates the pilots, channel response, and received signal in a $2 \times 1$ MIMO system. The delay-Doppler profile and system parameters used for the figure is given in Tables 1 and 2. The pilots placed in the delayDoppler grid have some space reserved around it, to account for the delay and Doppler spread of the channel. Each transmit and receive antenna pair sees a different channel with finite and nonoverlapping support, determined by the delay and Doppler spread of the channel ${ }^{14}$. Hence, the channel for all the transmit-receive antenna pairs

\begin{tabular}{ll} 
Table 4: Svstem parameters for 28 GHz svstem. & Value \\
\hline Parameter & 28 \\
\hline Carrier frequency $(\mathrm{GHz})$ & $(128,128)$ \\
Frame size $(M, N)$ & 78.125 \\
Subcarrier spacing $(\mathrm{kHz})$ & BPSK \\
Modulation scheme & $2 \times 2$ \\
MIMO configuration & 9 \\
\hline K factor $(\mathrm{dB})$ & 5 \\
No. of taps $(P)$ & 47 \\
\hline Maximum speed $(\mathrm{km} / \mathrm{h})$ &
\end{tabular}

can be simultaneously estimated using single MIMO-OTFS frame as described below.

The relation between the transmitted symbols from $p$ th transmit antenna and the received symbols from the $q$ th receive antenna can be written using (15) as

$$
\begin{aligned}
y_{q}[k, l]= & \sum_{m=0}^{M-1} \sum_{n=0}^{N-1} x_{p}[n, m] \frac{1}{M N} \\
& h_{w_{q p}}\left(\frac{k-n}{N T}, \frac{l-m}{M \Delta f}\right)+v_{q}[k, l] .
\end{aligned}
$$

Now, if the transmitted pilot from the pth antenna is given by

$$
\begin{aligned}
x_{p}[n, m] & =1 \text { if }(n, m)=\left(n_{p}, m_{p}\right) \\
& =0 \forall(n, m) \neq\left(n_{p}, m_{p}\right),
\end{aligned}
$$

the received signal at the $q$ th antenna will be

$y_{q}[k, l]=\frac{1}{M N} h_{w_{q p}}\left(\frac{k-n_{p}}{N T}, \frac{l-m_{p}}{M \Delta f}\right)+v_{q}[k, l]$.

Now, $\frac{1}{M N} h_{w_{q p}}\left(\frac{k}{N T}, \frac{l}{M \Delta f}\right)$ can be estimated from

(42), since $n_{p}$ and $m_{p}$ are known at the receiver a priori. From this, the equivalent channel matrix $\hat{\mathbf{H}}_{q p}$ can be obtained using the vectorized formulation of Sect. 3.3.

Observe that due to the convolutive nature of the input-output relation, the impulse at $(n, m)=\left(n_{p}, m_{p}\right)$ is spread by the channel only up to the maximum delay and Doppler spread of the channel. Thus, if the pilots are spaced sufficiently far apart, in the delay-Doppler domain, they can be received without interference. Hence, the channel responses corresponding to all the transmit-receive antenna pairs can be estimated simultaneously using single MIMO-OTFS frame. Figure 17 illustrates this for a $2 \times 1$ MIMO-OTFS system with $(M, N)=(32,32)$ at $4 \mathrm{~dB}$ SNR. The 
Transmitted OTFS symbols

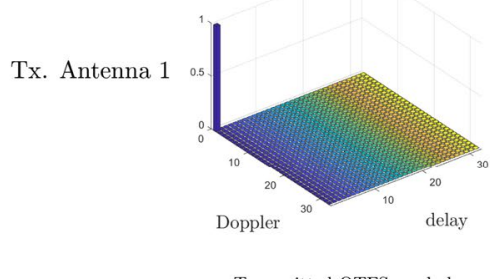

Transmitted OTFS symbols

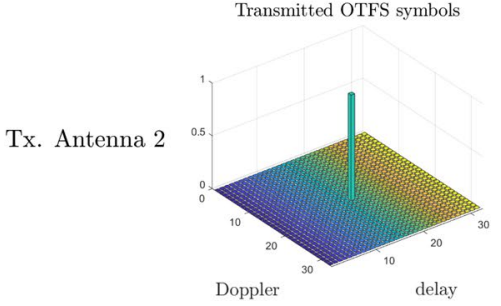

Channel

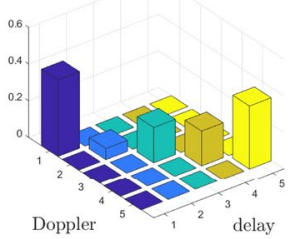

Channel

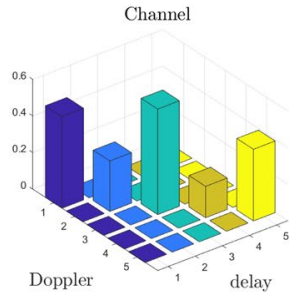

$2 \times 1$ system, $\mathrm{SNR}=4 \mathrm{~dB}$
Received OTFS symbols

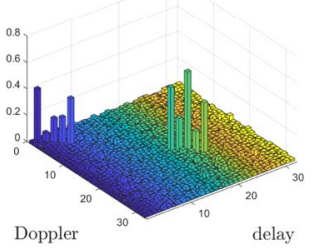

Figure 17: Illustration of pilots and channel response in delay-Doppler domain in a $2 \times 1$ system.

pilot corresponding to the first transmit antenna is placed at $\left(n_{1}, m_{1}\right)=(0,0)$ and the pilot corresponding to the second transmit antenna is placed at $\left(n_{2}, m_{2}\right)=(16,16)$ in the delay-Doppler domain. Also, note that the impulse responses at the receive antenna $h_{w_{11}}\left(\frac{k-n_{1}}{N T}, \frac{l-m_{1}}{M \Delta f}\right)$

and $h_{w_{12}}\left(\frac{k-n_{2}}{N T}, \frac{l-m_{2}}{M \Delta f}\right)$, corresponding to the

pilots transmitted from the first and the second transmit antennas, respectively, are non-overlapping at the receiver. Thus, they can be simultaneously estimated using a single pilot frame as described above.

The input-output relation in OTFS is convolutive in nature $(2 \mathrm{D}$ convolution of symbols and the channel response in delay-Doppler domain), whereas the input-output relation in any time-frequency modulation is multiplicative. This convolutive relation, along with the sparsity and time-invariance of delay-Doppler impulse response, greatly simplifies the channel estimation estimation. In Fig. 17, the pilot corresponding to the transmit antenna 1 gets convolved with the channel response of transmit antenna 1. Similarly, the pilot corresponding to the transmit antenna 2 convolves with the channel response of transmit antenna 2. At the receiver, the pilots are spread by the channel only to the extent of the support of each channel in the delay-Doppler domain. Since the channel in the delay-Doppler representation is sparse, the pilots can be received without interference. In contrast, in time-frequency domain, the received pilots overlap and span the entire time-frequency plane, making the channel estimation complex in doubly dispersive MIMO channels.

\subsection{Performance Results and Discussion}

In this subsection, the mean square error (MSE) of the estimated channel and the BER performance of MIMO-OTFS with the estimated channel are presented. The channel estimation scheme described in the previous subsection is used to

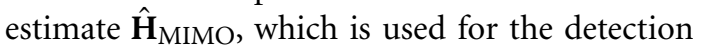
of the transmitted symbols using message passing algorithm. The simulations use Tables 1 and 2 for the delay-Doppler profile and the system parameters, respectively.

Figure 18 shows the normalized MSE $\left(\left\|\mathbf{H}_{\mathrm{MIMO}}-\hat{\mathbf{H}}_{\mathrm{MIMO}}\right\|_{F} /(M N)^{2}\right)$ of the estimated channel, as a function of the average SNR of the pilot frame, for a $2 \times 2$ MIMO-OTFS system. The system parameters used are as in Tables 1 and 2. From the figure, it can be observed that the normalized MSE decreases with the increase in the SNR of the pilot frame, as expected. Next, Fig. 19 shows the BER performance of the $2 \times 2$ MIMO-OTFS system with the estimated channel. From the figure, it is observed that the achieved BER performance with the estimated channel is very close to the performance with perfect CSIR. For example, at an SNR of $12.5 \mathrm{~dB}$, the achieved BER with the perfect knowledge of the channel is $2 \times 10^{-5}$, whereas the same BER is achieved at an SNR of $13 \mathrm{~dB}$, with the estimated channel. At the considered Doppler frequency as high as $1880 \mathrm{~Hz}$, channel estimation in the TF domain is very difficult and results in inaccurate estimation because of the rapid variations of the channel in 


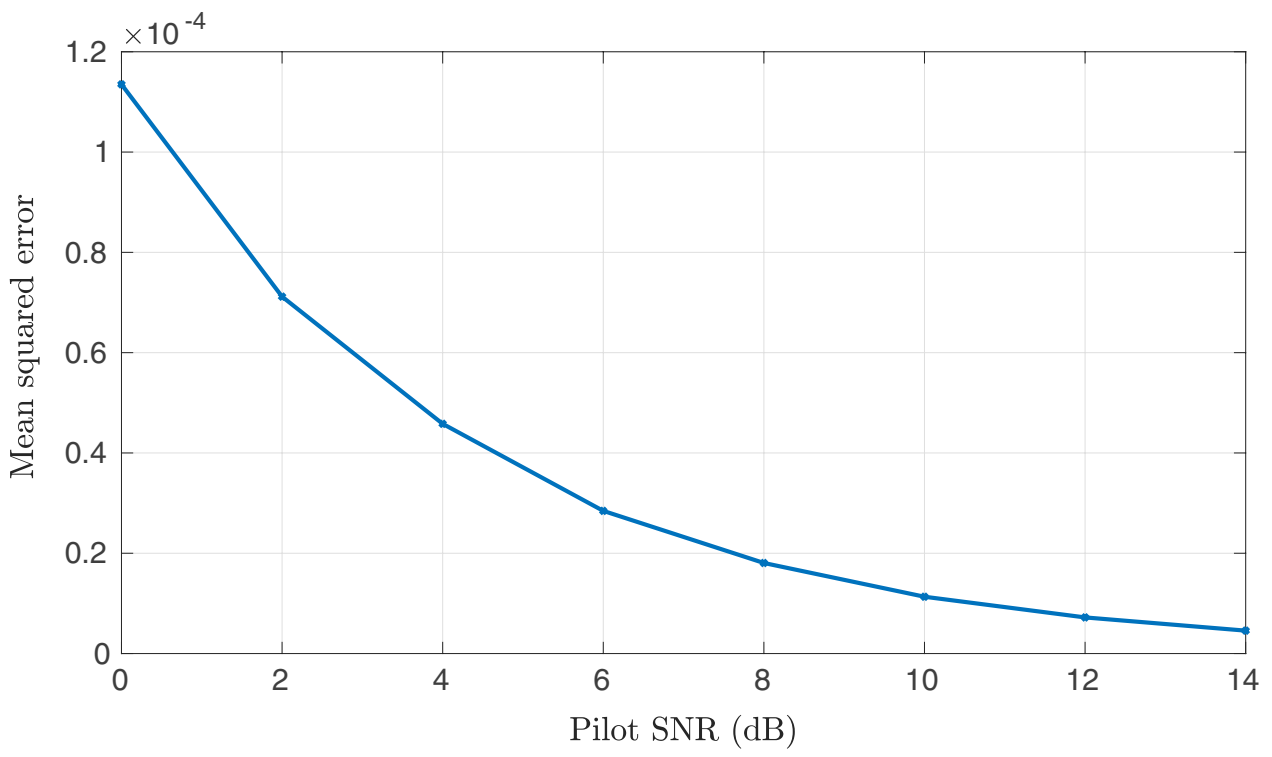

Figure 18: Mean square error of the estimated channel as a function of pilot SNR in a $2 \times 2$ MIMO system.

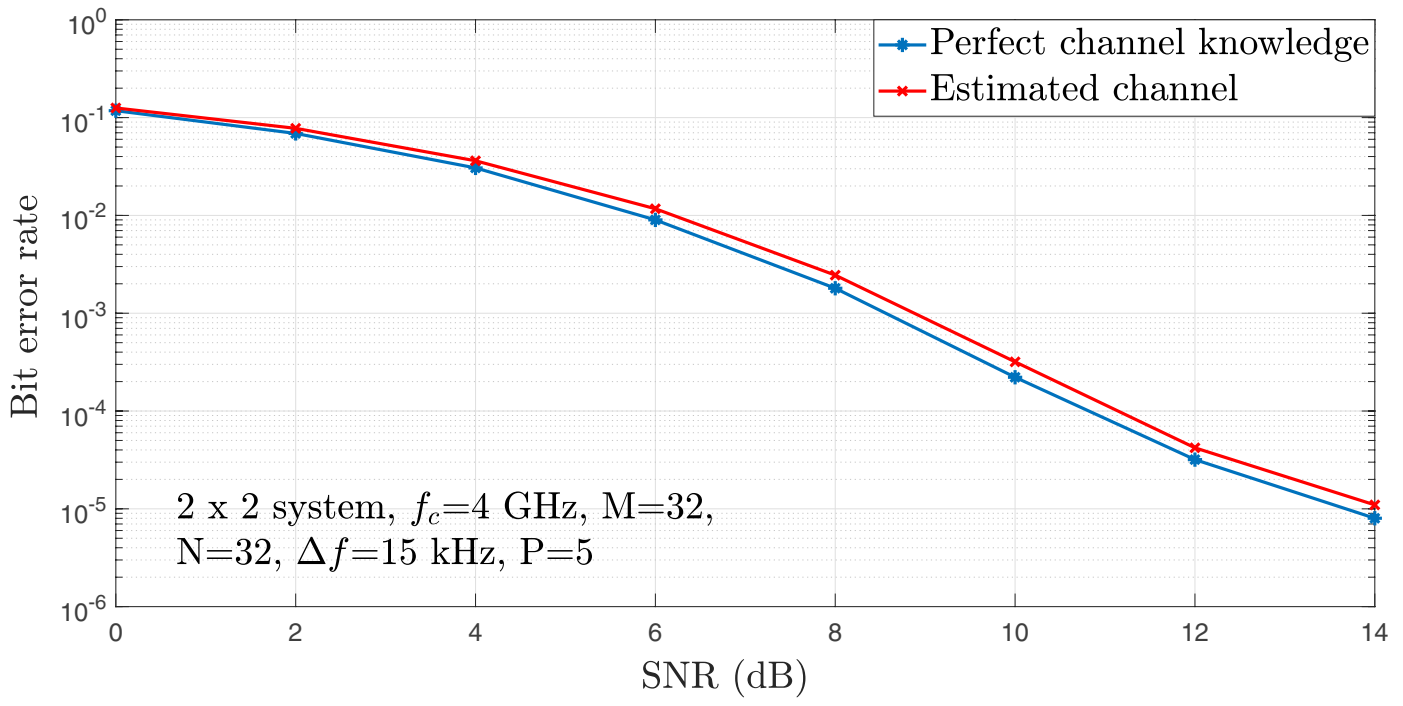

Figure 19: BER performance of MIMO-OTFS using the estimated channel in a $2 \times 2$ MIMO system.

time-frequency domain, whereas, the channel in the delay-Doppler representation is slowly varying and is time-invariant over a larger observation time. This, along with the $2 \mathrm{D}$ convolutive interaction of the symbols with the channel in the delay-Doppler domain, enables the proposed channel estimation for MIMO-OTFS to be simple and efficient.

\section{Conclusions}

We considered OTFS modulation, a 2D modulation scheme, where signaling is done in the delay-Doppler domain and can be implemented using existing multicarrier schemes using additional pre- and post-processing 2D transforms. It was shown to be a suitable modulation scheme for high-mobility use cases where the high Doppler shifts are encountered. While conventional modulation schemes such as OFDM fail to perform well in such highmobility scenarios due to Doppler-induced ICI, OTFS achieved significantly better performance. 
Considering a MIMO-OTFS system, we presented signal detection and channel estimation schemes and their performance. MIMO-OTFS was shown to achieve significantly better performance compared to MIMO-OFDM in highDoppler environments operating in $4 \mathrm{GHz}$ and $28 \mathrm{GHz}$ frequency bands. The sparse and slow time-variant nature of wireless channels in the delay-Doppler domain enabled simple and efficient channel estimation. The performance and implementation attributes of OTFS make it a promising modulation scheme for high-mobility use cases.

\section{Publisher's Note}

Springer Nature remains neutral with regard to jurisdictional claims in published maps and institutional affiliations.

Received: 20 December 2019 Accepted: 20 March 2020 Published online: 24 April 2020

\section{References}

1. Hattachi RE, Erfanian JJ (eds) (2015) 5G white paper. NGMN Alliance, Frankfurt a. M.

2. Jakes WC (1994) Microwave mobile communications. IEEE Press, New York

3. Goldsmith A (2005) Wireless communications. Cambridge University Press, Cambridge

4. Wang T, Proakis JG, Masry E, Zeidler JR (2006) Performance degradation of OFDM systems due to Doppler spreading. IEEE Trans Wireless Commun 5(6):1422-1432

5. Strohmer T, Beaver S (2003) Optimal OFDM design for time-frequency dispersive channels. IEEE Trans Commun 51(7):1111-1122

6. Han F-M, Zhang X-D (2007) Hexagonal multicarrier modulation: a robust transmission scheme for timefrequency dispersive channels. IEEE Trans Signal Process 55(5):1955-1961

7. Han F-M, Zhang X-D (2009) Wireless multicarrier digital transmission via Weyl-Heisenberg frames over timefrequency dispersive channels. IEEE Trans Commun 57(6):1721-1733

8. Sayeed AM, Aazhang B (1999) Joint multipath-Doppler diversity in mobile wireless communications. IEEE Trans Commun 47(1):123-132

9. Bhashyam S, Sayeed AM, Aazhang B (2000) Timeselective signaling and reception for communication over multipath fading channels. IEEE Trans Commun 48(1):83-94

10. Wornell GW (1996) Spread-response precoding for communication over fading channels. IEEE Trans Inform Theory 42:488-501
11. Hadani R, Rakib S, Tsatsanis M, Monk A, Goldsmith AJ, Molisch AF, Calderbank R (2017) Orthogonal time frequency space modulation. In: Proc. IEEE WCNC'2017, pp 1-7

12. Hadani R, Monk A (2018) OTFS: a new generation of modulation addressing the challenges of 5G. arXiv :1802.02623 ([cs.IT] 7 Feb 2018)

13. Hadani R, Rakib S, Kons S, Tsatsanis M, Monk A, Ibars C, Delfeld J, Hebron Y, Goldsmith AJ, Molisch AF, Calderbank R (2018) Orthogonal time frequency space modulation. arXiv:1808.00519v1 ([cs.IT] 1 Aug 2018)

14. Monk A, Hadani R, Tsatsanis M, Rakib S (2016) OTFSorthogonal time frequency space: a novel modulation technique meeting 5G high mobility and massive MIMO challenges. arXiv:1608.02993 ([cs.IT] 9 Aug 2016)

15. Hadani R, Rakib S, Molisch AF, Ibars C, Monk A, Tsatsanis M, Delfeld J, Goldsmith A, Calderbank R (Jun. 2017) Orthogonal time frequency space (OTFS) modulation for millimeter-wave communications systems. In: Proc. IEEE MTT-S Intl. Microwave Symp., pp 681-683

16. Surabhi GD, Chockalingam A (2019) OTFS modulation with phase noise in mmWave communications. In: Proc. IEEE VTC'2019-Spring

17. Li L, Wei H, Huang Y, Yao Y, Ling W, Chen G, Li P, Cai Y (2017) A simple two-stage equalizer with simplified orthogonal time frequency space modulation over rapidly time-varying channels. arXiv:1709.02505v1 ([cs.IT] 8 Sep 2017)

18. Raviteja P, Phan KT, Hong Y, Viterbo E (2018) Interference cancellation and iterative detection for orthogonal time frequency space modulation. IEEE Trans Wireless Commun 17(10):6501-6515

19. Reyhani AR, Farhang A, Ji M, Chen R-R, Farhang-Boroujeny B (2018) Analysis of discrete-time MIMO OFDMbased orthogonal time frequency space modulation. In: Proc. IEEE ICC'18, pp 1-6

20. Dean T, Chowdhury M, Goldsmith A (2017) A new modulation technique for Doppler compensation in frequency-dispersive channels. In: Proc. IEEE PIMRC'2017

21. Farhang A, Reyhani AR, Doyle LE, Farhang-Boroujeny B (2018) Low complexity modem structure for OFDMbased orthogonal time frequency space modulation. IEEE Wireless Commun Lett 7(3):344-347

22. Murali KR, Chockalingam A (2018) On OTFS modulation for high-Doppler fading channels. In: Proc. ITA'2018, San Diego

23. Surabhi GD, Augustine RM, Chockalingam A (2019) On the diversity of uncoded OTFS modulation in doubly-dispersive channels. IEEE Trans Wireless Commun 18(6):3049-3063

24. Raviteja P, Hong Y, Viterbo E, Biglieri E (2018) Practical pulse-shaping waveforms for reduced-cyclic-prefix OTFS. IEEE Trans Veh Tech 68(1):957-961

25. Nimr A, Chafii M, Matthe M, Fettweis G (2018) Extended GFDM framework: OTFS and GFDM comparison. In: Proc. IEEE GLOBECOM'2018 
26. Ramachandran MK, Chockalingam A (2018) MIMOOTFS in high-Doppler fading channels: signal detection and channel estimation. In: Proc. IEEE GLOBECOM'2018. Abu Dhabi

27. Rakib S, Hadani R (2017) Multiple access in wireless telecommunications system for high-mobility applications, US Patent No. US9722741B1

28. Khammammetti V, Mohammed SK (2019) OTFS based multiple-access in high Doppler and delay spread wireless channels. IEEE Trans Wireless Commun 8(2):528-531

29. Surabhi GD, Augustine RM, Chockalingam A (2019) Multiple access in the delay-Doppler domain using OTFS modulation. arXiv:1902.03415v1 ([cs.IT] 9 Feb 2019)

30. Augustine RM, Chockalingam A (2019) Interleaved timefrequency multiple access using OTFS modulation. In: Proc. IEEE VTC'2019-Fall

31. Raviteja P, Phan KT, Hong Y (2019) Embedded pilotaided channel estimation for OTFS in delay-Doppler channels. IEEE Trans Veh Tech 68(5):4906-4917

32. Surabhi GD, Augustine RM, Chockalingam A (2019) Peak-to-average power of OTFS modulation. IEEE Commun Lett 23(6):999-1002

33. Tiwari S, Das SS (2019) Circularly pulse shaped orthogonal time frequency space modulation. Electron Lett. arXiv:1910.10457 ([cs.IT] 23 Oct 2019)

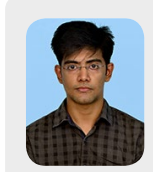

M. K. Ramachandran received the B.Tech. degree in electronics and communication engineering from the College of Engineering Trivandrum, India, in 2015, and the M.Tech degree in electrical communication engineering from the Indian Institute of Science, Bengaluru, India, in 2018. He is currently with the Wireless R\&D Division, Qualcomm India Private Ltd., Bengaluru. His interests include low-complexity signal demodulation and channel estimation schemes for wireless waveforms.

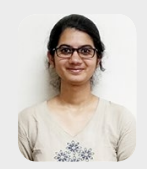

G. D. Surabhi received the B.E. degree in electronics and communication engineering from Siddaganga Institute of Technology, Tumkur, India, in 2014. She is currently pursuing the Ph.D. degree with the Department of Electrical Communication Engineering, Indian Institute of Science, Bangalore, India. Her current research interests include mmWave MIMO communication systems and orthogonal time-frequency space modulation.

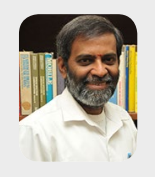

A. Chockalingam (S'92-M'93-SM'98) was born in Rajapalayam, Tamil Nadu, India. He received the B.E. (Honors) degree in electronics and communication engineering from the P. S. G. College of Technology, Coimbatore, India, in 1984, the M.Tech. degree in electronics and electrical communications engineering (with specialization in satellite communications)
34. Augustine RM, Surabhi GD, Chockalingam A (2019) Space-time coded OTFS modulation in high-Doppler channels. In: Proc. IEEE VTC’2019-Spring

35. Tiwari S, Das SS, Rangamgari V (2019) Low complexity LMMSE receiver for OTFS. IEEE Commun Lett. (preprint in IEEE Xplore)

36. Surabhi GD, Chockalingam A (2019) Low-complexity linear equalization for OTFS modulation. IEEE Commun Lett. (preprint in IEEE Xplore)

37. Samimi MK, Maccartney GR, Sun S, Rappaport TS (2016) $28 \mathrm{GHz}$ millimeter-wave ultrawideband smallscale fading models in wireless channels. In: Proc. IEEE VTC'2016-Spring, pp 1-6

38. Liu P, Springer A (2015) Space shift keying for LOS communication at mmWave frequencies. IEEE Wireless Commun Lett 4(2):121-124

39. Bohagen F, Orten P, Oien G (2007) Design of optimal high-rank line-of-sight MIMO channels. IEEE Trans Wireless Commun 6(4):1420-1425

40. Fish A, Gurevich S, Hadani R, Sayeed AM, Schwartz O (2013) Delay-Doppler channel estimation in almost linear complexity. IEEE Trans Inf Theory 59(11):7632-7644

41. Hlawatsch F, Matz G (2011) Wireless communications over rapidly time-varying channels. Academic Press, New York

from the Indian Institute of Technology, Kharagpur, India, in 1985, and the Ph.D. degree in electrical communication engineering (ECE) from the Indian Institute of Science (IISc), Bangalore, India, in 1993. During 1986 to 1993, he worked with the Transmission R\&D division of the Indian Telephone Industries Limited, Bangalore. From December 1993 to May 1996, he was a Postdoctoral Fellow and an Assistant Project Scientist at the Department of Electrical and Computer Engineering, University of California, San Diego. From May 1996 to December 1998, he served Qualcomm, Inc., San Diego, CA, as a Staff Engineer/Manager in the systems engineering group. In December 1998, he joined the faculty of the Department of ECE, IISc, Bangalore, India, where he is a Professor, working in the area of wireless communications and networking. Dr. Chockalingam is a recipient of the Swarnajayanti Fellowship from the Department of Science and Technology, Government of India. He served as an Associate Editor of the IEEE TRANSACTIONS ON VEHICULAR TECHNOLOGY, and as an Editor of the IEEE TRANSACTIONS ON WIRELESS COMMUNICATIONS. He served as a Guest Editor for the IEEE JOURNAL ON SELECTED AREAS IN COMMUNICATIONS (Special Issue on Multiuser Detection for Advanced Communication Systems and Networks), and for the IEEE JOURNAL OF SELECTED TOPICS IN SIGNAL PROCESSING (Special Issue on Soft Detection on Wireless Transmission). He is a Fellow of the Indian National Academy of Engineering, the National Academy of Sciences, India, the Indian National Science Academy, and the Indian Academy of Sciences. 